INRA Prod. Anim., 2008, 21 (3), 277-290

\title{
Les fibres dans l'alimentation des truies gestantes : effets sur la nutrition, le comportement, les performances et les rejets dans l'environnement
}

\author{
F.-X. PHILIPPE', V. REMIENCE', J.-Y. DOURMAD ${ }^{3}$, J.-F. CABARAUX'1, M. VANDENHEEDE ${ }^{1}$, B. NICKS \\ 1 Université de Liège, Département des Productions Animales, Boulevard de Colonster 20, Bât. B43, 4000 Liège, Belgique \\ 2 Centre Wallon de Recherches Agronomiques, Département Productions et Nutrition Animales, \\ Rue de Liroux 8, 5030 Gembloux, Belgique \\ 3 INRA, UMR1079, Systèmes d'Elevage, Nutrition Animale et Humaine, F-35000, Rennes, France \\ Courriel :fxphilippe@ulg.ac.be
}

\begin{abstract}
Actuellement, les élevages doivent non seulement intégrer des facteurs économiques de rentabilité, mais également des contraintes en termes de bien-être animal et de respect de l'environnement. La législation européenne impose par exemple la présence de fibres dans l'alimentation des truies gestantes, afin d'apaiser leur faim et de satisfaire leur besoin de mastication. Cette synthèse évalue l'impact réel d'une telle mesure sur le comportement des animaux, les performances zootechniques et les rejets dans l'environnement, tout en décrivant les mécanismes digestifs sous-jacents.
\end{abstract}

La conduite alimentaire des truies a une influence déterminante sur leurs performances de reproduction. Le défi consiste à obtenir en fin de gestation un niveau de réserves corporelles suffisant tout en évitant une prise de poids trop importante. Un excès de poids et/ou d'adiposité à la mise bas peut en effet entraîner des problèmes de locomotion et aussi des dystocies éventuellement compliquées par des mammites, avec des répercussions négatives sur le taux de survie des porcelets et la longévité du troupeau (Dourmad et al 1994, Le Cozler et al 1999).

L'état corporel des truies à la mise bas interfère également avec le niveau d'ingestion pendant la lactation : les truies les plus grasses ont un appétit plus faible, ce qui entraîne une forte mobilisation de leurs réserves et l'apparition du phénomène de «truie accordéon» (Dourmad 1991). D'autre part, une réduction excessive des réserves corporelles durant cette période peut s'accompagner de retours en oestrus retardés et d'un taux de conception plus faible (Prunier et al 1993, Dourmad et al 1994, Eissen et al 2003, Quesnel et al 2007). Afin d'assurer l'adéquation entre les besoins, faibles en gestation et élevés en lactation, et les apports alimentaires, le schéma d'alimentation généralement appliqué en élevage est composé d'une phase de restriction alimentaire en gestation suivie d'une phase de distribution libérale en lactation (Ramonet et al 1997).

Le rationnement pendant la gestation favorise cependant l'apparition de comportements stéréotypés associés à une motivation alimentaire non satisfaite (Terlouw et al 1991). Lorsque les truies sont logées en groupe, la frustration alimentaire occasionne en outre une augmentation des agressions (Svendsen et Svendsen 1997). Plusieurs études ont montré que l'ajout de fibres alimentaires dans la ration permet de réduire ces comportements stéréotypés et d'agression (Brouns et al 1994a, Ramonet et al 1997, 1999, Robert et al 1997, Whittaker et al 2000) qui sont souvent considérés comme des indicateurs de mal-être des animaux (Dantzer 1986, Broom 1988). La légis- lation européenne, par la directive 2001/88/CE, impose d'ailleurs la mise à disposition des truies gestantes d'un aliment riche en fibres.

Le présent article a pour objectif de passer en revue les conséquences de l'incorporation de fibres dans la ration des truies gestantes sur la physiologie digestive, les comportements et les performances des animaux ainsi que sur le bilan environnemental lié à cette production.

\section{1 / Les fibres alimentaires}

Différentes définitions du terme «fibres alimentaires» sont proposées, selon que l'on se place du point de vue botanique, chimique ou physiologique. Pour les nutritionnistes, les fibres alimentaires regroupent les composants de l'aliment qui résistent à la digestion par les enzymes du tube digestif des mammifères (Bach Knudsen 2001). Ces composants sont principalement représentés par la lignine et les polysaccharides complexes des parois végétales, à 
Figure 1. Répartition des différents types de fibres en fonction de leur solubilité et de la méthode d'analyse (TDF, total dietary fibre ; NSP, non-starch polysaccharides ; NDF, neutral detergent fibre ; $A D F$, acid detergent fibre ; $A D L$, acid detergent lignine), d'après Le Goff (2001).

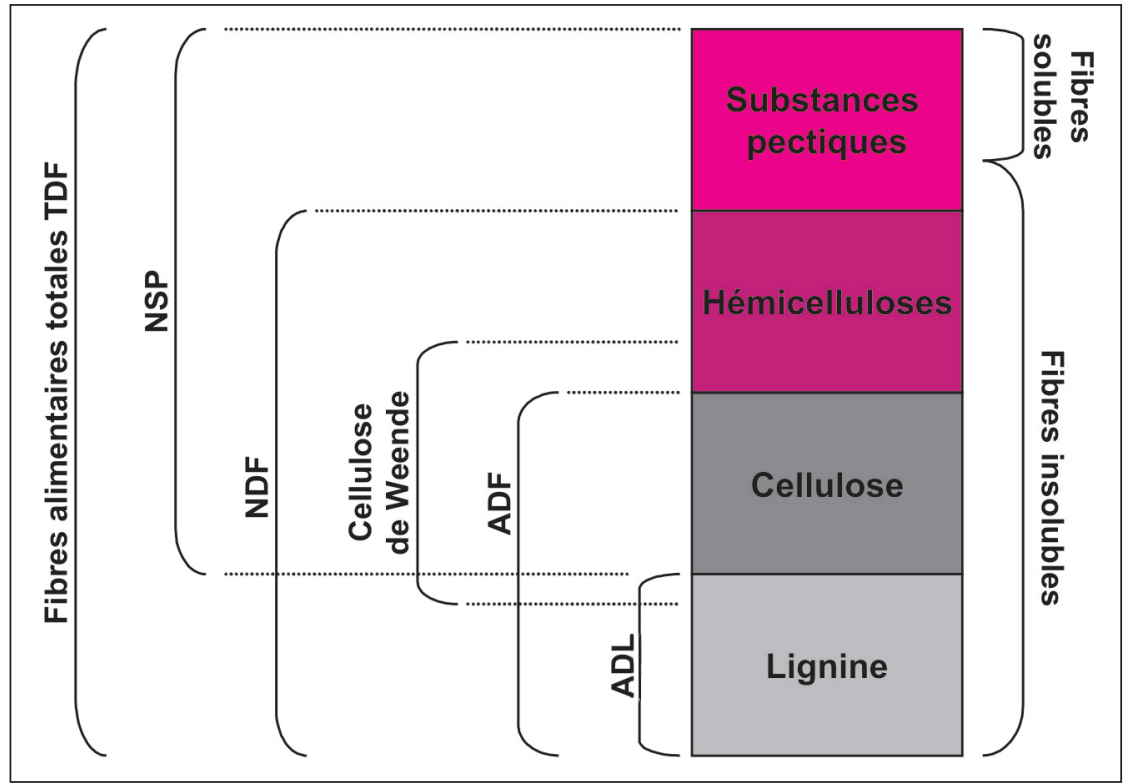

savoir la cellulose, les hémicelluloses et les substances pectiques (figure 1).

La cellulose est un polymère linéaire constitué de milliers d'unités glucose liées en $\beta(1,4)$. Les hémicelluloses sont des polymères de longueur variable formés d'un squelette composé principalement de xylose, mannose et/ou glucose, et portant des chaînes latérales en nombre et composition variables. La cellulose et les hémicelluloses sont incrustées de lignine, substance non-glucidique hautement indigestible. Compte tenu de leur insolubilité dans l'eau, l'ensemble formé par la cellulose, les hémicelluloses et la lignine est désigné sous la dénomination de fibres insolubles.

Les fibres hydrosolubles sont représentées par les substances pectiques, formées d'un squelette d'unités acide galacturonique dans lequel sont dispersées des unités rhamnose et portant des chaînes à base d'arabinose et galactose (Thibault et al 1994). Les gommes et mucilages, composés cytoplasmiques également solubles, leur sont apparentés du fait de leurs propriétés nutritionnelles analogues.

La dénomination de polysaccharides non amylacés (NSP, Non-Starch Polysaccharides) correspond à l'ensemble formé par les fibres solubles et insolubles, à l'exception de la lignine.

Différentes procédures permettent d'analyser le contenu en fibres des matières premières (Bach Knudsen 2001), dont les principales sont présentées ci-dessous.

La méthode séquentielle de Van Soest (1967) mesure les fractions de fibres insolubles dans :

- un détergent neutre (NDF, Neutral Detergent Fibre), à savoir la cellulose, les hémicelluloses et la lignine ;

- un détergent acide (ADF, Acid Detergent Fibre), soit la cellulose et la

- l'acide sulfurique (ADL, Acid Detergent Lignine), la lignine uniquement. lignine ; et lignine (ADL), d'après Sauvant et al (2004).
La méthode de Weende détermine le pourcentage de cellulose brute après double hydrolyse à chaud de l'aliment, d'abord avec une solution acide, puis avec une solution basique. La cellulose de Weende correspond à la totalité de la cellulose vraie, ainsi qu'à une petite fraction d'hémicelluloses et de lignine résistant à la double hydrolyse.

La méthode de Prosky (Prosky et al 1988) quantifie les fibres alimentaires totales (TDF, Total Dietary Fibre). Elle consiste en une élimination enzymatique successive de l'amidon et des protéines, suivie d'une précipitation alcoolique des fibres. Les TDF représentent les NSP, la lignine ainsi qu'une partie de l'amidon cytoplasmique résistant à la dégradation enzymatique. Le résultat de ces différentes méthodes d'analyse est également présenté sur la figure 1.

Les principales sources de fibres utilisées en alimentation porcine, ainsi que leurs caractéristiques sont présentées dans le tableau 1.

\section{2 / Effets sur la nutrition}

\section{1 / Comportement alimentaire et niveau d'ingestion}

L'incorporation de fibres alimentaires dans la ration des truies gestantes diminue leur motivation alimentaire. Une augmentation du temps d'alimentation combinée à une diminution de la vitesse d'ingestion a ainsi été relevée avec des niveaux croissants de fibres. L'ampleur des modifications est égale-

Tableau 1. Principales matières premières riches en fibres et leurs teneurs, en $\% d u$ produit sec, en cellulose (CB), neutral detergent fibre (NDF), acid detergent fibre (ADF)

\begin{tabular}{lcccc}
\hline & CB & NDF & ADF & ADL \\
\hline Paille de blé & 41,8 & 78,9 & 50,1 & 8,2 \\
Coques de soja & 38,3 & 63,1 & 45,2 & 2,3 \\
Cosses d'avoine & 29,0 & 73,5 & 35,3 & 6,9 \\
Luzerne déhydratée & 28,4 & 46,1 & 32,6 & 8,3 \\
Tourteaux de tournesol & 28,7 & 46,3 & 33,0 & 11,4 \\
Pulpes de betterave déshydratées & 19,4 & 45,5 & 23,1 & 2,1 \\
Graine de tournesol & 16,7 & 31,0 & 20,1 & 6,1 \\
Drèches d'orge de brasserie & 16,6 & 57,5 & 22,2 & 5,9 \\
Avoine & 14,0 & 35,4 & 16,2 & 2,7 \\
Tourteaux de colza & 13,9 & 31,7 & 20,6 & 9,9 \\
Son de blé & 10,6 & 45,5 & 13,7 & 3,9 \\
Corn gluten feed & 8,5 & 38,4 & 10,0 & 1,3 \\
Drèches de maïs & 8,3 & 39,2 & 13,9 & 2,3 \\
Drèches de blé & 7,9 & 23,7 & 10,8 & 4,6 \\
Pois & 6,0 & 14,3 & 7,1 & 0,4 \\
\hline
\end{tabular}


ment fonction de la nature de l'aliment fibreux. En augmentant le taux d'incorporation de cosses d'avoine de 0 à $50 \%$, la vitesse d'ingestion passe de $136 \mathrm{~g} / \mathrm{min}$ à $83 \mathrm{~g} / \mathrm{min}$ (Mroz et al 1986). En augmentant la teneur en pulpes de betterave de 0 à $30 \%$, la durée d'ingestion passe de 12 à $18 \mathrm{~min}$ (Rijnen et al 2003). A même apport énergétique, le remplacement d'aliments à base de céréales par des aliments enrichis en fibres peut augmenter jusqu'à 3 à 5 fois les durées des repas (Brouns et al 1994a, 1997) et diminuer par deux la vitesse d'ingestion (Guillemet et al 2006). La consommation peut également être plus fractionnée, avec davantage d'interruptions pendant les repas. Par exemple, avec un aliment à $31 \%$ de NDF, le nombre d'interruptions est de 0,93 par repas comparé à 0,01 avec un aliment à $17 \%$ de NDF (Guillemet et al 2006).

Ces différences de comportement alimentaire peuvent tout d'abord s'expliquer par la moindre appétence des fibres. En effet, lors de tests en double choix, les truies montrent une nette préférence pour un aliment à base de céréales plutôt que pour un aliment riche en fibres (Guillemet et al 2007a). Cette faible appétence des fibres se traduit également par davantage de refus alimentaires (Cerneau et al 1997, Paboeuf et al 2000).

La diminution de la motivation alimentaire peut aussi résulter de la meilleure aptitude des fibres à rassasier les truies. A même apport énergétique, une ration enrichie en fibres occupe un plus grand volume, ce qui favorise la satiété par distension gastro-intestinale (Martin et Edwards 1994, Moran et al 1999). De plus, par leur caractère généralement plus grossier et leur capacité de rétention d'eau, les fibres augmentent le temps consacré à la préhension et à la mastication (Ramonet et al 1999, 2000a). Les sécrétions de salive et de sucs gastriques sont ainsi stimulées (Zebrowska et al 1983, Mosenthin et al 1994, Brouns et al 1997) venant encore accroître le contenu de l'estomac et réduire la sensation de faim. Le ralentissement de la vidange gastrique observé avec certaines fibres prolongerait cet effet, tout comme le retard des pics post-prandiaux en glucose et insuline qui en découle (Ramonet et al 2000a, de Leeuw et al 2005). Les mécanismes de rétrocontrôle régulant la prise alimentaire sont ainsi retardés. En outre, la production d'Acides Gras Volatils $(\mathrm{AGV})$ associée à la fermentation des fibres dans le côlon constitue une source retardée d'énergie qui pourrait aussi limi- ter la motivation alimentaire à long terme (de Leeuw et al 2005).

Parmi les différentes sources de fibres, les pulpes de betterave semblent avoir un effet plus marqué. Ainsi, la quantité d'aliment consommé lors d'une distribution ad libitum est moindre lorsqu'il contient des pulpes de betterave (Brouns et al 1995, Whittaker et al 2000, van der Peet-Schwering et al 2004) alors que les cosses d'avoine, les sons de riz et de blé et les drèches de brasseries ne semblent pas modifier les niveaux d'ingestion (tableau 2, Brouns et al 1995). La grande capacité de rétention d'eau des pulpes de betterave (Bertin et al 1988) expliquerait principalement cette différence. D'autre part, il semblerait que des niveaux élevés en fibres soient nécessaires pour obtenir un effet significatif (Brouns et al 1995, Paboeuf et al 2000, Whittaker et al 2000, van der Peet-Schwering et al 2004). Ainsi, une incorporation de pulpes de betterave, limitée à $15 \%$ de la ration, ne réduit pas la vitesse d'ingestion (Cerneau et al 1997). D'après van der Peet-Schwering et al (2004), le niveau de consommation volontaire ne diminue significativement que lorsque la teneur en pulpes de betterave atteint $45 \%$. A titre indicatif, le niveau d'ingestion volontaire observé avec des aliments contenant entre 40 et $65 \%$ de pulpes de betterave est de l'ordre de $4 \mathrm{~kg} / \mathrm{j}$ (Brouns et al 1995, Whittaker et al 2000, van der Peet-Schwering et al 2004), alors qu'il est voisin de $7 \mathrm{~kg} / \mathrm{j}$ avec un aliment standard (Bergeron et al 2000) ou des aliments contenant des sources de fibres autres que des pulpes de betterave (Brouns et al 1995).

Tableau 2. Quantités d'aliment et d'Energie Digestible (ED) ingérées par des truies gestantes nourries ad libitum au moyen d'aliments enrichis en fibres de sources et taux d'incorporation différents (NDF, neutral detergent fibre ; MS, matière sèche), d'après Brouns et al (1995).

\begin{tabular}{lcccc}
\hline & \multicolumn{3}{c}{ Quantité ingérée } & \multicolumn{2}{c}{ Contenu de l'aliment } \\
\cline { 2 - 5 } $\begin{array}{l}\text { Source de fibres } \\
\text { et taux d'incorporation }\end{array}$ & $\begin{array}{c}\text { Aliment } \\
\text { (kg/j) }\end{array}$ & $\begin{array}{c}\text { ED } \\
\text { (MJ/j) }\end{array}$ & $\begin{array}{c}\text { NDF } \\
\text { (g/kg de MS) }\end{array}$ & $\begin{array}{c}\text { ED } \\
\text { (MJ/kg MS) }\end{array}$ \\
\cline { 2 - 5 } & 6,4 & 57,0 & 404,8 & 10,3 \\
Paille d'orge (36\%) & 7,7 & 79,0 & 361,3 & 11,8 \\
Cosses d'avoine (37\%) & 6,8 & 70,1 & 278,9 & 13,2 \\
Drèches de brasserie (46\%) & 7,6 & 70,8 & 373,7 & 10,5 \\
Son de riz (61\%) & 7,1 & 72,9 & 329,2 & 11,9 \\
Son de blé (67\%) & 5,0 & 58,3 & 338,5 & 13,8 \\
Pulpes de betterave (40\%) & 4,1 & 45,8 & 353,3 & 13,3 \\
Pulpes de betterave (50\%) & 4,5 & 38,8 & 352,4 & 13,1 \\
Pulpes de betterave (58\%) & 3,5 & 364,8 & 12,8 \\
Pulpes de betterave (65\%) & 3,0 & 32,1 & 36 & \\
\hline
\end{tabular}

Une trop forte teneur en fibres des rations pourrait par ailleurs empêcher l'animal de consommer une quantité suffisante d'énergie, par incapacité physique d'ingérer plus d'aliment alors que les apports restent inférieurs aux besoins (Robert et al 1997, Whittaker et al 1998, Meunier-Salaün et al 2001, Holt et al 2006). En alimentation ad libitum, Brouns et al (1995) constatent une perte de lard dorsal associée à une diminution de l'ingestion volontaire d'énergie digestible qui passe de 58 à $32 \mathrm{MJ} /$ jour avec l'incorporation de respectivement 40 et $65 \%$ de pulpes de betterave dans la ration (tableau 2). Le caractère volumineux de la ration agirait à court terme sur la réduction de la sensation de faim (Robert et al 1997, Meunier-Salaün et al 2001, Holt et al 2006), le besoin en énergie finissant par stimuler l'appétit à plus long terme (Martin et Edwards 1994).

Enfin, l'effet sur l'appétit évoluerait avec le temps, l'aversion initiale aux régimes riches en fibres pouvant être passagère (Paboeuf et al 2000). Des mécanismes d'adaptation métabolique (Brouns et al 1994b) et l'accroissement de la capacité du tube digestif permettraient en outre une augmentation progressive de l'ingestion volontaire (Whittaker et al 2000).

Concernant l'ingestion d'eau, certains auteurs ont observé une réduction de la consommation chez les truies gestantes recevant des rations enrichies en fibres (Farmer et al 1996, Ramonet et al 1999, Robert et al 2000). A titre d'exemciée à un régime à base d'avoine et cosple, la consommation quotidienne asso- 
ses d'avoine a été de 11,4 L par truie pour $20,6 \mathrm{~L}$ avec un régime à base de maïs (Robert et al 2000). Cet effet est attribué à une réduction générale de la motivation alimentaire en présence de fibres. D'autres auteurs n'ont pas relevé de différence significative, principalement en raison d'une grande variabilité individuelle dans les consommations (Pollmann et al 1979, Levasseur et Courboulay 2001, Massé et al 2003, Fernandez 2006). Ces différents résultats restent cependant difficilement interprétables en raison de gaspillage possible au niveau de l'abreuvoir (Levasseur et Courboulay 2001).

\section{2 / Transit digestif}

Différents facteurs influencent la vitesse de vidange de l'estomac, en particulier, la teneur en fibres de la ration, le volume des repas, le mode de présentation des aliments et la taille des particules (Potkins et al 1991, Guerin et al 2001).

L'effet des fibres sur la vidange de l'estomac est sujet à controverse. S'il semble établi que les fibres ralentissent la vidange de la fraction liquide du contenu gastrique (Guerin et al 2001, Miquel et al 2001), leur effet sur la fraction solide est plus équivoque avec une durée de rétention plus courte (Potkins et al 1991), inchangée (Miquel et al 2001, Partanen et al 2007, Wilfart 2007) ou plus longue (Guérin et al 2001). Les résultats observés semblent principalement dépendre du type de fibres utilisées. Ainsi, l'incorporation de gomme de guar ou de pectine, s'accompagne d'une accélération de la vidange gastrique, alors que celle de son et d'avoine n'a pas d'effet significatif (Potkins et al 1991). En revanche, avec des pulpes de betterave, la rétention gastrique est prolongée (Guérin et al 2001, Miquel et al 2001). En comparant une ration standard (11\% NDF) à une ration enrichie en pulpes de betterave (27\% NDF), Guérin et al (2001) obtiennent un temps de demi-vidange de respectivement 78 et $112 \mathrm{~min}$. L'effet des pulpes est attribué à leur aptitude à augmenter la capacité de liaison à l'eau et la viscosité des digesta, ce qui rend le passage du pylore plus difficile (Guérin et al 2001). Par ailleurs, la présence dans l'intestin d'AGV issus des fermentations coliques inhibe la motricité et la vidange de l'estomac (Cuche et al 2000).

Au niveau des intestins, la vitesse de transit est principalement déterminée par le temps de présence des digesta dans le caecum-côlon (Latymer et al 1990). Les résultats expérimentaux montrent un transit accéléré (Partanen et al 2007), ralenti (Wilfart et al 2007) ou non modifié (Potkins et al 1991), en fonction de la nature des fibres. De grandes variations entre individus et stades physiologiques ont également été relevées (Latymer et al 1990, Le Goff et al 2002b, Jørgensen et al 2007).

Les fibres insolubles à taux de lignification élevé étant peu digestibles, elles se retrouvent en grande quantité dans le côlon où, elles conservent leur propriété de liaison à l'eau. Par leur volume, elles agissent alors physiquement sur la motilité du côlon (Cherbut et al 1988, Potkins et al 1991, Le Goff et al 2002b, Serena et al 2007, Wilfart et al 2007). De même, la production d'AGV dans le côlon, stimule les contractions de sa paroi et y augmente la vitesse de passage des digesta (Salvador et Cherbut 1992).

L'origine botanique des fibres semble aussi déterminante. Ainsi, les sons de maïs et de blé, bien que de compositions semblables en fibres solubles et insolubles, n'ont pas le même effet sur la vitesse du transit avec un temps moyen de rétention dans le tube digestif de respectivement 78,1 et 68,6 h (Le Goff et al 2002b). L'accessibilité différente des fibres à la dégradation microbienne expliquerait cette différence.

La vitesse de transit est aussi influencée par la taille et la morphologie des différents segments du tractus digestif, paramètres sur lesquels les fibres ont également un impact.

\section{3 / Morphologie du tube di- gestif}

La présence de fibres dans la ration augmente le poids du tube digestif (Anguita et al 2007). L'effet est le plus marqué au niveau de l'estomac et surtout du côlon, l'intestin grêle semblant peu concerné (Jørgensen et al 1996, Libao-Mercado et al 2007, Serena et al 2007). C'est le volume plus élevé de ce type de ration qui favorise physiquement le développement des organes digestifs (Whittaker et al 2000). L'augmentation du poids des truies gestantes nourries avec des rations enrichies en fibres est ainsi partiellement attribuable au développement du tube digestif et à l'accroissement de son contenu (Ramonet et al 1999).
Chez les truies, on observe également une modification de la muqueuse du côlon avec une augmentation de la profondeur et de la surface des cryptes (Serena et al 2007). Chez le porc charcutier, les effets s'étendent jusqu'à l'iléon et au jéjunum avec augmentation des villosités intestinales et accélération du turnover cellulaire (Jin et al 1994, Libao-Mercado et al 2007). C'est avec des fibres facilement fermentescibles, riches en cellulose et hémicelluloses et pauvres en lignine, que l'on relève les différences les plus significatives (Libao-Mercado et al 2007, Serena et al 2007). Ces modifications seraient attribuables à la présence de molécules issues des fermentations, telles les $\mathrm{AGV}$, ou de la fragmentation des fibres, tels l'acide galacturonique, agissant par l'intermédiaire d'hormones, de neuropeptides ou d'autres agents. On avance également un effet direct des fibres sur la muqueuse via leurs propriétés physico-chimiques, comme la viscosité par exemple (Plaisancie et al 1996, Deplancke et Gaskins 2001, Fukunaga et al 2003, Libao-Mercado et al 2007, Serena et al 2007).

\section{4 / Digestibilité}

La digestibilité des nutriments est affectée par la présence de fibres dans les rations. De nombreux auteurs ont ainsi observé une diminution de la digestibilité de la matière sèche, de la matière organique, des protéines brutes, de la matière grasse et de l'énergie (Mroz et al 1986, Jørgensen et al 1996, Ramonet et al 1999, 2000b, Noblet et Le Goff 2000, Olesen et al 2001, Le Goff et al 2002a, Holt et al 2006, Partanen et al 2007). Ces effets ont été constatés avec de nombreuses sources de fibres, mais dans des proportions variables en fonction de leur origine botanique. Ainsi, à teneur en énergie brute identique, Le Goff et al (2002a) obtiennent des coefficients de digestibilité fécale apparente de l'énergie de $90 \%, 87 \%$ et $85 \%$ avec des aliments enrichis respectivement en pulpes de betterave (16\% NDF), en son de maïs (20\% NDF) et en son de blé $(16 \%$ NDF), soit des valeurs plus faibles que pour un aliment standard à base de céréales $(11 \% \mathrm{NDF})$ qui présente une digestibilité de l'énergie de $93 \%$. Les résultats de digestibilité des fibres elles-mêmes sont plus contradictoires (Mroz et al 1986, Yan et al 1995, Jørgensen et al 1996, Canh et al 1997, Ramonet et al 2000b, Le Goff et al 2002a, Serena et al 2007). Pour des taux d'incorporation en pulpes de betterave de 15 et $42 \%$, la digestibilité des NDF est respectivement de 75 et $85 \%$ 
(Ramonet et al 2000b, Le Goff et al 2002a). Avec des rations contenant de 0 à $50 \%$ de cosses d'avoines, la valeur passe respectivement de 67 à $30 \%$ (Mroz et al 1986).

La digestibilité dépend fortement du degré de lignification des fibres. Une lignification importante limite l'accessibilité des enzymes digestives et bactériennes aux composants cellulaires (Bach Knudsen 1997). L'accélération du transit liée à l'augmentation des sécrétions digestives a tendance également à diminuer la digestibilité (Satchithanandan et al 1990, Jørgensen et al 1996). Le phénomène peut cependant être partiellement compensé par les modifications de taille et de la morphologie du tube digestif, principalement par celles s'opérant au niveau du côlon (Jørgensen et al 1996). D'autres facteurs, comme la solubilité des fibres, l'interaction avec les autres nutriments, le niveau d'alimentation, le poids vif et le stade physiologique des animaux peuvent interférer également avec la digestibilité des divers composants de la ration (Jørgensen et al 1996, Noblet et Le Goff 2000, Rijnen et al 2001, Le Goff et al 2002a, Serena et al 2007).

\section{5 / Métabolisme azoté}

L'incorporation de fibres entraîne une modification de la répartition des pertes d'azote par l'organisme, sans que la fraction retenue par les animaux ne soit modifiée. Ainsi, la part excrétée dans les urines est diminuée alors que celle excrétée dans les matières fécales est augmentée (Canh et al 1997, 1998b, Ramonet et al 2000b, Olesen et al 2001, Le Goff et al 2002a, Zervas et Zijltra 2002, Lynch et al 2007, 2008). Ces modifications s'expliquent par la présence dans le gros intestin d'une plus grande quantité de glucides fermentescibles qui servent de source d'énergie pour la flore microbienne et stimulent ainsi la croissance bactérienne (Wenk 2001, Partanen et al 2007). Cela induit un plus grand transfert d'urée depuis le sang vers la lumière de l'intestin (Low 1985). L'azote est alors utilisé pour la synthèse de protéines microbiennes qui sont finalement excrétées dans les fèces. Ce transfert d'azote de l'urine vers les matières fécales conduit à une réduction de la digestibilité apparente des protéines.

\section{6 / Métabolisme énergétique}

Avec les fibres, une plus grande partie de la production d'énergie digestible est associée aux fermentations qui s'opèrent dans le côlon (Shi et Noblet
1993, Ramonet et al 2000b). Chez la truie, les produits de ces fermentations, principalement les $\mathrm{AGV}$, peuvent représenter plus de $30 \%$ des besoins énergétiques d'entretien (Varel et Yen 1997). L'efficacité énergétique de ces transformations au sein du côlon est cependant plus faible qu'une absorption par l'intestin grêle des monosaccharides issus des hydrates de carbone, une partie de l'énergie étant perdue sous forme de méthane $\left(\mathrm{CH}_{4}\right)$, d'hydrogène $\left(\mathrm{H}_{2}\right)$ et de chaleur de fermentation (Le Goff et al 2002a). Une approche biochimique de l'utilisation des fibres montre d'ailleurs que le rendement énergétique obtenu lors des fermentations est inférieur à celui obtenu lors de la digestion enzymatique (Dierick et al 1989, Le Goff 2001).

L'impact des fibres sur les pertes d'énergie dans l'urine semble peu important (Ramonet et al 2000b, Olesen et al 2001, Le Goff et al 2002a,

Figure 2. Relations entre la production de méthane entérique $\left(\mathrm{CH}_{4}\right)$ et la quantité de fibres digestibles ingérées par les truies, dNDF (digestible neutral detergent fibre) et dADF (digestible acid detergent fibre), d'après Jørgensen et al (1996), Ramonet et al (2000b), Olesen et al (2001), Rijnen et al (2001), Le Goff et al (2002a), Jørgensen (2007), Jørgensen et al (2007), Warpechowski et al (2007).

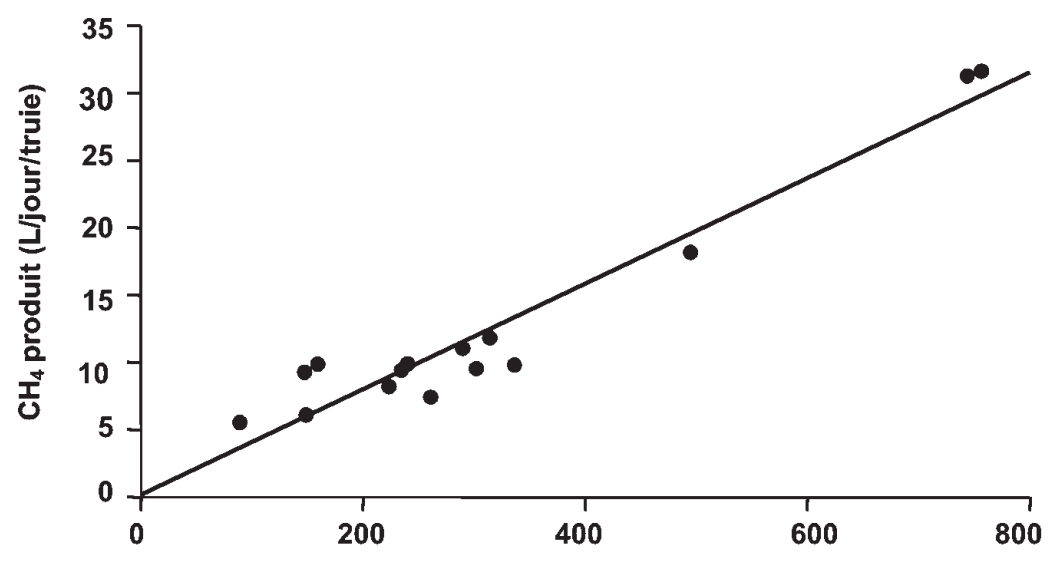

dNDF ingérés (g/jour/truie)

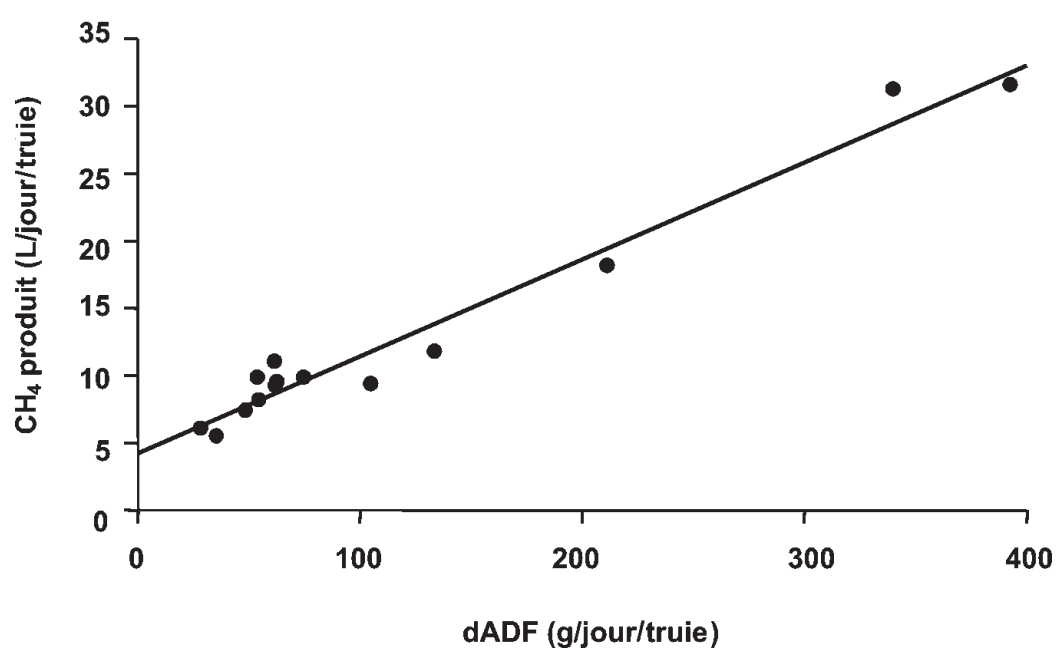


avec $\mathrm{CH}_{4}$, la production de méthane en $\mathrm{L} / \mathrm{j}$ par truie, dNDF, la quantité de NDF digestibles ingérée en $\mathrm{kg} / \mathrm{j}$ par truie et dADF, la quantité de ADF digestibles ingérée en $\mathrm{kg} / \mathrm{j}$ par truie. Par exemple, l'ingestion de $250 \mathrm{~g}$ de dNDF correspond à une production de $10 \mathrm{~L}$ de $\mathrm{CH}_{4}$, soit une perte énergétique d'environ $400 \mathrm{~kJ}$. Cette valeur est proche de celle avancée par Noblet et al (2003), avec une perte d'énergie sous forme de gaz d'environ $350 \mathrm{~kJ}$ pour l'ingestion de $250 \mathrm{~g}$ de paroi végétale. Outre la teneur en fibres, la variabilité des résultats provient également de leur origine botanique, structure, solubilité et fermentescibilité.

Bien que Le Goff et al (2002a), n'observent pas d'accroissement significatif de la production de chaleur avec différentes sources de fibres, il est généralement admis qu'elles augmentent les pertes d'énergie sous cette forme (Jørgensen et al 1996, Ramonet et al $2000 \mathrm{~b}$, Olesen et al 2001). Cela a pour conséquence une réduction de l'énergie retenue par les truies, avec diminution des dépôts adipeux mais sans impact sur la rétention de protéines (Ramonet et al 2000b). Par ailleurs, il a été démontré que, dans un environnement froid, cette augmentation de la production de chaleur était efficacement utilisée pour la thermorégulation (Noblet et al 1989, Jørgensen et al 1996, Ramonet et al 2000b). De plus, en modifiant le comportement des truies, les rations riches en fibres peuvent diminuer les pertes d'énergie liées à l'activité physique, les truies étant moins souvent debout (Robert et al 1993, Brouns et al 1994a, Noblet et al 1994, Ramonet et al 1999) et ce malgré un temps d'alimentation plus long. Une plus grande part de l'énergie est alors disponible pour les dépôts corporels. L'utilisation d'un système énergie nette tel qu'il est proposé dans les tables INRA-AFZ (Sauvant et al 2004) permet de prendre en compte les différences de rendement énergétique des divers nutriments et sécurise ainsi la formulation des régimes à teneur élevée en fibres.

Chez la truie, le rendement de l'énergie provenant des fibres est plus élevé que chez le porc en croissance, grâce à la meilleure efficacité de la dégradation des fibres. Cela peut s'expliquer par la taille plus grande du tube digestif, la vitesse de transit plus lente et le nombre de bactéries cellulolytiques plus important (Varel et Yen 1997, Le Goff et Noblet 2001, Le Goff et al 2002a, 2002b). Par contre, la capacité intrinsèque de la flore microbienne à dégra- der les fibres ne serait pas meilleure chez la truie que chez le porc en croissance (Le Goff et al 2003).

\section{3 / Conséquences sur les comportements non- alimentaires}

Si l'activité alimentaire des truies (temps quotidien d'alimentation) est influencée par la teneur en fibres des rations, celle-ci interfère également avec la fréquence observée des comportements stéréotypés et d'agression entre animaux (Danielsen et Vestergaard 2001) ainsi qu'avec leur niveau général d'activité (Robert et al 1993, Ramonet et al 1999, Paboeuf et al 2000).

\section{1 / Fréquence des comporte- ments stéréotypés}

Il est courant d'observer des activités stéréotypées chez des truies gestantes rationnées et qui n'ont pas ou peu accès à un substrat manipulable. Par définition, ces activités sont des séquences comportementales accomplies de façon répétitive dans un ordre fixe et sans fonction apparente (Meunier-Salaün et al 2001). Elles peuvent être interprétées comme une dégradation des conditions de bien-être de l'animal (Wiepkema et al 1983). Les comportements stéréotypés sont plus fréquents dans la période qui suit immédiatement le repas et semblent refléter une motivation alimentaire non satisfaite. Les consommations excessives d'eau qui ne sont plus sous le contrôle des mécanismes normaux de régulation sont également considérées comme des stéréotypies (Terlouw et al 1991).

Les stéréotypies, qu'elles soient non alimentaires ou associées à la potomanie, sont moins fréquentes avec des rations enrichies en fibres. Par exemple, la distribution d'un aliment contenant $15 \%$ de pulpes de betterave diminue les activités orales non alimentaires par rapport à un aliment témoin voisin sur le plan énergétique (Cerneau et al 1997). Des résultats similaires ont été obtenus avec un régime enrichi en son de blé et pulpes de betterave (Courboulay et Gaudre 2002). De même, Paboeuf et al (2000) obtiennent moins de léchage de l'auge avec des aliments contenant $20 \%$ de son de blé et $20 \%$ de pulpes de betterave. Un apport de paille au sol permet aussi de réduire les activités orales répétitives avec ou sans utilisation d'un support (Fraser 1975, Whittaker et al 1999).

La réduction des activités stéréotypées associée à des rations enrichies en fibres semble liée à leur encombrement plus important mais pourrait aussi être due à l'augmentation du temps consacré à l'ingestion (Meunier-Salaün et al 2001). De deux rations enrichies en fibres et assurant un même apport d'énergie, celle présentant l'encombrement le plus important (à base de cosses d'avoine) s'est montrée plus efficace à réduire la fréquence des stéréotypies que l'autre à base de son de blé et rafles de maïs. L'incorporation de fibres dans les rations des truies leur permet ainsi de satisfaire leur motivation alimentaire en consommant une quantité d'aliment plus importante sans qu'elles n'ingèrent davantage de nutriments. Mais si le taux de fibres dans l'aliment devient excessif, la consommation d'aliment deviendra limitante, et la quantité de nutriments consommés, et notamment l'énergie, ne suffira pas à couvrir les besoins des animaux. Or, un régime avec un contenu inadéquat en nutriments augmente les stéréotypies pré-nourrissage (Robert et al 1997).

L'effet de régimes fibreux sur la réduction des stéréotypies évolue avec le rang de portée. Ces régimes semblent plus efficaces chez les jeunes truies (en particulier les truies de seconde portée), ceci étant sans doute lié au fait que les stéréotypies deviennent graduellement plus rigides et fréquentes au cours du temps (Dantzer 1986, Robert et al 1993, Meunier-Salaün et al 2001). Chez des truies en quatrième gestation nourries avec un régime riche en fibres, la fréquence des activités orales non alimentaires avec support est diminuée mais pas celle des activités sans support, ces dernières apparaissant ainsi moins facilement réversibles (Bergeron et al 2000). Compte tenu de ces observations, il conviendrait de distribuer aux truies des régimes fibreux dès leur première gestation (Paboeuf et al 2000).

La réduction des activités stéréotypées est plus forte lorsque le taux d'incorporation de matériaux fibreux se situe autour de $300 \mathrm{~g}$ de NDF/kg par rapport à un niveau plus bas (Robert et al 1993, 1997, Ramonet et al 1999).

La fréquence des repas interfère aussi avec les résultats. D'après Holt et al (2006) une distribution en deux repas est plus efficace qu'un seul repas. Mais 
d'après Robert et al (2002), des cochettes nourries une fois par jour réalisent moins de comportements stéréotypés autour du repas que celles nourries deux fois par jour.

\section{2 / Comportements agressifs}

Une alimentation rationnée peut amener une compétition alimentaire se traduisant par des comportements agressifs chez les truies nourries en groupe avec accès restreint à la nourriture (Meunier-Salaün et al 2001). L'apport d'aliment fibreux semble pouvoir réduire, dans certains cas, la fréquence des agressions, en particulier les morsures de vulve chez des truies élevées en groupe (van Putten et van de Burgwal 1990, Whittaker et al 1999). Ponctuellement, une diminution de la sévérité des agressions a été relevée le premier jour suivant l'introduction des truies dans un groupe dynamique lorsqu'une alimentation rationnée au distributeur automatique de concentré était remplacée par une distribution ad libitum d'un aliment riche en fibres (Whittaker et al 1995). En comparant un régime témoin ( $88 \%$ d'orge) à deux régimes enrichis en fibres, une diminution de la fréquence des agressions n'a été relevée qu'avec un régime à base de $50 \%$ de pulpes de betterave, alors qu'aucune différence n'a été observée avec un régime contenant $20 \%$ de cosses d'avoine, $15 \%$ de son de blé et $15 \%$ d'herbe déshydratée, les apports en énergie nette étant identiques dans les trois groupes (Danielsen et Vestergaard 2001).

\section{3 / Activité motrice}

Les truies recevant un aliment fibreux sont, en général, moins actives que celles nourries avec un aliment standard (Bergeron et al 2000, Paboeuf et al 2000, van der Peet-Schewering et al 2003b, de Leeuw et al 2005). Cette moindre activité associée à une augmentation du temps passé couché pourrait être interprétée comme une mesure de la satisfaction alimentaire (MeunierSalaün et al 2001).

Le temps passé en position debout est ainsi réduit de 25\% (291 vs 394 $\mathrm{min} /$ jour) lorsque les truies sont nourries avec un aliment fibreux $(39 \%$ de NDF) en comparaison à un aliment témoin (13,5\% de NDF, Ramonet et al 1999). Cette réduction est particulièrement marquée au cours de la période pré-prandiale (Paboeuf et al 2000). La durée de la position debout est par ailleurs étroitement corrélée à la fréquence des stéréotypies (Ramonet et al
1999). Le nombre de changements de posture est également diminué (Robert et al 1993).

L'effet peut dépendre de la source des fibres. Il est en effet plus marqué avec un régime constitué à $41 \%$ d'avoine et $53 \%$ de cosses d'avoine comparativement à un régime constitué à $43 \%$ de son de blé et $53 \%$ de rafles de maïs (Robert et al 1993). D'autre part, la réduction d'activité est plus importante chez les truies plus âgées, par exemple, chez des truies en seconde portée, comparées à des cochettes (Robert et al 1993).

\section{4 / Conséquences sur les performances}

\section{1 / Performances des truies}

\section{a) Variations de poids et d'épaisseur de lard dorsal}

Plusieurs études ont montré que, en comparaison avec des rations standard à apport en énergie digestible identique, les rations enrichies en fibres apportées aux truies gestantes ne modifient ni leur gain de poids pendant la gestation (Farmer et al 1996, Cerneau et al 1997, Paboeuf et al 2000, Guillemet et al 2007b), ni leur gain de lard dorsal (Farmer et al 1996, Cerneau et al 1997, Paboeuf et al 2000), quels rangs de portée.

D'autres auteurs ont cependant constaté une prise de poids plus importante avec un aliment fibreux (Mroz et al 1986, Whittaker et al 2000, Danielsen et Verstergaard 2001). Les causes sont à rechercher dans une sous-estimation de la valeur énergétique de la ration (Mroz et al 1986) et/ou dans l'accroissement $\mathrm{du}$ volume du tube digestif et la réque soient les sources des fibres et les

plétion intestinale (Danielsen et Verstergaard 2001). Au moment de la mise bas, le passage à l'aliment de lactation standard provoque d'ailleurs une rapide perte de poids, signe de l'adaptation du tube digestif au régime plus concentré (Vestergaard et Danielsen 1998, Danielsen et Verstergaard 2001, van der Peet-Schwering et al 2003a). La réduction de la taille des organes digestifs et leur réplétion correspondrait à environ 5-6 kg (Vestergaard et Danielsen 1998, van der PeetSchwering et al 2003a).

A l'inverse des résultats rapportés cidessus, des gains de poids et d'épaisseur du lard dorsal insuffisants durant la gestation ont pu être relevés et attribués à des apports trop faibles en énergie nette, la part d'énergie brute convertie en $\mathrm{CH}_{4}$ n'ayant pas été suffisamment prise en compte (Matte et al 1994, van der Peet-Schwering et al 2003a, Guillemet et al 2007b).

Les truies qui ont consommé des rations riches en fibres durant la gestation présentent généralement un niveau d'ingestion volontaire plus élevé durant la lactation (tableau 3, Matte et al 1994, Farmer et al 1996, Danielsen et Vestergaard 2001, Courboulay et Gaudré 2002, van der Peet-Schwering et al 2003a). Cela s'explique en partie par l'accroissement de la taille et de la capacité des organes digestifs, lié au caractère plus volumineux des rations fibreuses. Cet effet a cependant tendance à s'estomper avec le temps. A titre d'exemple, un aliment de gestation composé à $49 \%$ de cosses d'avoine modifie la quantité ingérée au cours des quatre semaines de lactation de respectivement $+16 \%,+14 \%,+9 \%$ et - 6\% (Farmer et al 1996). Un effet positif limité à la première semaine de lactation a également été relevé par Guillemet et al (2006).

Tableau 3. Comparaison des consommations alimentaires en lactation (en $\mathrm{kg} / \mathrm{j}$ ) en fonction du type d'aliment apporté durant la gestation.

\begin{tabular}{cccl}
\hline $\begin{array}{c}\text { Aliment } \\
\text { témoin }\end{array}$ & $\begin{array}{c}\text { Aliment } \\
\text { fibreux }\end{array}$ & $\begin{array}{c}\text { Différence } \\
\mathbf{( \% )}\end{array}$ & Références \\
\hline 4,95 & $5,15^{\mathrm{a}}$ & $+4,0$ & Farmer et al (1996) \\
6,07 & $6,34^{\mathrm{b}}$ & $+4,4$ & Courboulay et Gaudré (2002) \\
5,91 & $6,23^{\mathrm{c}}$ & $+5,4$ & Danielsen et Vestergaard (2001) \\
5,41 & $5,98^{\mathrm{d}}$ & $+10,5$ & van der Peet-Schwering et al (2003a) \\
\hline
\end{tabular}

Source de fibres de l'aliment de gestation :

a : son d'avoine $(49 \%)$

b : pulpes de betterave $(22 \%)$ et son de blé $(22 \%)$

c : pulpes de betterave $(50 \%)$

d : pulpes de betterave $(38 \%)$ 
L'impact sur les consommations en lactation semble également dépendre de la source de fibres. Un aliment contenant $50 \%$ de pulpes de betterave augmente les consommations de 5\% par rapport à un aliment à base de céréales alors qu'un aliment composé à $20 \%$ de cosses d'avoine, $15 \%$ de son de blé et $15 \%$ d'herbe déshydratée est sans effet (Danielsen et Vestergaard 2001). De même, un régime à base d'avoine $(41 \%)$ et cosses d'avoine (53\%) présente un effet plus marqué qu'un régime à base de son de blé $(43 \%)$ et rafles de maïs (53\%, Matte et al 1994).

Que les rations soient ou non enrichies en fibres, les niveaux d'ingestion sont fortement tributaires de l'état corporel des truies, celles ayant le moins de réserves adipeuses à la mise bas consommant le plus d'aliment au cours de la lactation suivante (Dourmad 1991, Matte et al 1994, Le Cozler et al 1999, Danielsen et Vestergaard 2001). Notons cependant que différents auteurs n'ont pas montré de modifications significatives de la consommation des truies en lactation suite à l'apport de fibres en gestation (Honeyman et Zimmerman 1990, Whittaker et al 2000), même avec un état corporel plus faible à la mise bas (Guillemet et al 2007b).

Malgré une augmentation de l'ingestion en lactation, l'état des truies au sevrage (poids vif, épaisseur de lard dorsal) n'est généralement pas affecté par une alimentation enrichie en fibres durant la gestation (Matte et al 1994, Farmer et al 1996, Danielsen et Vestergaard 2001, van der PeetSchwering et al 2003a, Guillemet et al 2007b). Des pertes de poids plus élevées en lactation ont parfois été signalées mais sans diminution accrue de l'épaisseur du lard dorsal, (Whittaker et al 2000, Guillemet et al 2006). Elles sont dans ce cas attribuables à l'adaptation du tube digestif à l'aliment de lactation, comme discuté ci-dessus.

En conclusion, les rations de gestation riches en fibres ne pénalisent pas l'état des truies à la mise bas. Elles peuvent entraîner une légère augmentation de l'ingestion durant la lactation, probablement due à l'accroissement de la capacité du tube digestif, mais n'interfèrent pas avec l'état corporel des truies au sevrage.

\section{b) Déroulement de la mise bas}

Le bon déroulement de la mise bas a une grande importance sur la survie des porcelets en péri-partum (van Dijk et al
2005). Peu d'études ont été entreprises pour évaluer l'impact de rations de gestation riches en fibres sur ce paramètre. Aucun effet significatif ni sur la durée du part, ni sur celle de la gestation, l'intervalle entre les mises bas et les naissances des porcelets n'a été relevé par Guillemet et al (2007b). En revanche, d'autres auteurs ont constaté des réductions de la durée du part allant de 9 à $29 \%$ chez des truies qui recevaient un régime enrichi en fibres (Morgenthum et Bolduan 1988, KurcmanPrsedpelska 1989, Bilkei 1990). Le type, la source et le taux d'incorporation des fibres, ainsi que le type génétique des truies pourraient être à l'origine de ces différences.

\section{c) Retour en oestrus}

Aucun impact négatif de rations de gestation riches en fibres n'a été relaté à propos des taux de conception ou des intervalles entre le sevrage et le retour en oestrus, quels que soient la source et le contenu en fibres, le rang de portée des truies et le nombre de cycles reproductifs étudiés (Matte et al 1994, Paboeuf et al 2000, Whittaker et al 2000, van der Peet-Schwering et al 2003a, Guillemet et al 2007b).

\section{2 / Prolificité des truies et poids des porcelets}

Peu de différences ressortent globalement des comparaisons effectuées entre les performances des portées de truies nourries avec un aliment standard ou enrichi en fibres.

A propos des nombres de porcelets nés totaux et nés vivants, de nombreuses études concluent à une absence d'effet, quels que soit le rang de portée des truies ou le nombre de cycles de reproduction étudiés (Matte et al 1994, Farmer et al 1996, Cerneau et al 1997, Paboeuf et al 2000, Whittaker et al 2000, Danielsen et Vestergaard 2001, Courboulay et Gaudré 2002, Guillemet et al 2007b). Certaines études, en revanche, plaident pour un effet positif des rations de gestation riches en fibres, que celles-ci soient à base de cosses d'avoine (40\% NDF, Mroz et al 1986), de corn gluten feed (34\% NDF, Honeyman et Zimmerman 1990) ou de pulpes de betterave (47\% NSP, van der Peet-Schwering et al 2003a). Une étude relate quant à elle un effet négatif d'un aliment contenant des coques de soja (Holt et al 2006). Le moment d'introduction des fibres dans le cycle de reproduction pourrait avoir son importance (Mroz et al 1986, van der PeetSchwering et al 2003a). D'après
Bergner (1981), augmenter le contenu en fibres avant insémination favoriserait l'implantation et la survie des embryons par réduction de l'absorption d'amines toxiques par le gros intestin.

Le poids individuel des porcelets à la naissance et le poids des portées ne semblent pas dépendants de la teneur en fibres des rations (Farmer et al 1996, Cerneau et al 1997, Paboeuf et al 2000, Whittaker et al 2000, Guillemet et al 2007b). Certaines études rapportent cependant un poids individuel plus faible à la naissance avec des aliments riches en fibres (Courboulay et Gaudré 2002, van der Peet-Schwering et al 2003a). La source des fibres influence également les résultats. Par exemple, un poids moyen significativement moins élevé $(1,47 \mathrm{~kg})$ a été relevé avec un aliment composé à $50 \%$ de pulpes de betterave comparé à ceux obtenus avec des aliments soit à base de cosses d'avoine, son de blé et herbe déshydratée $(1,65 \mathrm{~kg})$ ou à base d'orge $(1,62 \mathrm{~kg})$, toutes les rations étant équilibrées en énergie nette (Danielsen et Vestergaard 2001). Le rang de portée des truies pourrait également interagir avec la teneur en fibres des rations. C'est ainsi qu'en première gestation, le poids à la naissance est inférieur avec un aliment à base de cosses d'avoine comparé à un aliment à base de maïs alors qu'en deuxième gestation, la tendance est inversée (Matte et al 1994). Outre la source de fibres et le rang de portée, des variabilités dans les consommations alimentaires ou une mauvaise évaluation de l'énergie nette ingérée peuvent donc également expliquer des différences de résultats. L'énergie ingérée durant la gestation, principalement durant le dernier mois, est un facteur important conditionnant le poids des porcelets à la naissance, la prise de poids fotale en fin de gestation étant directement fonction du niveau d'ingestion maternelle (Close et al 1984, Close et Cole 1986).

Au sevrage, le nombre et le poids des porcelets sont peu influencés par la présence de fibres dans la ration des truies gestantes, comme observé dans de nombreuses études (Honeyman et Zimmerman 1990, Matte et al 1994, Farmer et al 1996, Paboeuf et al 2000, Whittaker et al 2000, Danielsen et Vestergaard 2001, van der PeetSchwering et al 2003a, Holt et al 2006). Lorsqu'un effet positif est observé, il est attribué à une consommation supérieure en début de lactation combinée à une production laitière plus importante (Mroz et al 1986, Guillemet 
et al 2007b). Néanmoins, l'augmentation de l'ingestion des truies en lactation ne s'accompagne pas toujours d'une amélioration des performances des porcelets au sevrage (Matte et al 1994, Farmer et al 1996, Danielsen et Vestergaard 2001, Courboulay et Gaudré 2002, van der Peet-Schwering et al 2003a). Des consommations supérieures en lactation sont parfois liées à de moindres réserves corporelles à la mise bas (Matte et al 1994, van der Peet-Schwering et al 2003a). L'ingestion supplémentaire est alors utilisée pour limiter les pertes corporelles des truies mais sans effet bénéfique sur le poids des porcelets.

En conclusion, il ressort de la majorité des études menées qu'il n'y a pas à craindre de diminution des performances de reproduction suite à l'incorporation de fibres dans les rations de gestation, tant que des apports énergétiques suffisants sont assurés. Des effets soit positifs, soit négatifs, mais toujours d'ampleur limitée, ont parfois été rapportés en envisageant l'implication de critères secondaires tels que le rang de portée des truies, la source, le taux et le moment d'introduction des fibres. Des différences dans les types génétiques étudiés ou dans les conditions d'élevage peuvent également expliquer en partie la variabilité des résultats (Cerneau et al 1997).

\section{5 / Conséquences sur les rejets}

\section{1 / Déjections et effluents}

L'incorporation de fibres dans les rations de truies gestantes augmente la quantité de matières fécales produites (Mroz et al 1996, Robert et al 2000, Massé et al 2003, Fernandez 2006). A titre d'exemple, des truies nourries avec un aliment à base d'avoine $(40 \%)$ et de cosses d'avoine (50\%) produisent 6 fois plus de fèces que d'autres recevant un aliment standard à base de maïs $(9,1 v s$ 1,5 kg par jour, Robert et al 2000).

La teneur en MS des matières fécales diminue chez les truies recevant les régimes riches en fibres $(\mathrm{Mroz}$ et al 1986, Robert et al 2000, Fernandez 2006). La teneur des fèces en azote est également augmentée en raison d'une part de la plus faible digestibilité des protéines des matières premières riches en fibres et d'autre part de l'accroissement du transfert dans le gros intestin d'urée plasmatique vers les protéines bactériennes émises dans les fèces
(Canh et al 1997, Ramonet et al 2000b, Olesen et al 2001, Le Goff et al 2002a, Zervas et Zijltra 2002, Lynch et al 2007). La synthèse de protéines bactériennes est d'autant plus importante que les fibres sont solubles (Bindelle et al 2007). Par ailleurs, l'ajout de fibres s'accompagne d'un accroissement de la teneur en AGV des fèces, ce qui entraîne une diminution du $\mathrm{pH}$, d'autant plus marquée que les fibres sont fortement fermentescibles (Canh et al 1997, 1998a, 1998b, Hansen et al 2007, Lynch et al 2007).

Ces modifications des caractéristiques des matières fécales sont attribuables à l'action combinée de plusieurs facteurs comme l'accroissement de la flore bactérienne, la présence de fibres non digérées dans les fèces, la diminution de la digestibilité des autres nutriments et la capacité de rétention d'eau des fibres. La quantité de matières fécales produites et leur contenu en eau sont d'autant plus importants que les fibres sont peu fermentescibles, ce qui est notamment le cas de la paille de blé (Yan et al 1995, Fernandez 2006). Par contre, avec les pulpes de betterave, fortement fermentescibles, l'effet est plus marqué sur le $\mathrm{pH}$ qui est diminué via la production d'AGV, et particulièrement d'acétate (Canh et al 1998a, 1998b, Hansen et al 2007).

L'ingestion de fibres s'accompagne également d'une diminution de la production d'urine par les truies (Mroz et al 1996, Robert et al 2000, Massé et al 2003, Fernandez et al 2006). Par exemple, un contenu croissant en cosses d'avoine de 0 à $50 \%$ diminue la production d'urine qui passe de $17,3 \mathrm{~kg}$ à $6,3 \mathrm{~kg} / \mathrm{j}$ (Mroz et al 1986). Cette réduction s'explique principalement par le contenu en eau plus élevé des matières fécales (Mroz et al 1986, Robert et al 2000, Fernandez et al 2006). Une moindre consommation d'eau, parfois observée avec des rations riches en fibres, pourrait aussi contribuer aux différences observées (Farmer et al 1996, Robert et al 2000). Les rejets d'azote par l'urine sont également diminués suite à une utilisation d'une fraction de l'azote de l'urée pour la synthèse des protéines par la flore du côlon. Le ratio entre l'azote urinaire et l'azote fécal est ainsi diminué (Canh et al 1997, Hansen et al 2007, Lynch et al 2007). Chez le porc charcutier, ces ratios sont respectivement de $2,4,1,3$ et 1,0 avec des aliments à base de céréales, de coques de soja et de pulpes de betterave (Zervas et Zijltra 2002). Le $\mathrm{pH}$ de l'urine peut également être modifié avec les fibres mais cet effet est plutôt dû à un changement dans la balance électrolytique des régimes plutôt qu'à un effet des fibres per se (Patience et al 1987, Canh et al 1997).

L'augmentation de la quantité de matières fécales produites étant compensée par la diminution des rejets d'urine, le poids d'effluent n'est pas affecté par les rations riches en fibres, avec des valeurs moyennes de $20 \mathrm{~kg}$ de lisier produit par truie et par jour (Robert et al 2000, Levasseur et Courboulay 2001, Massé et al 2003). Le volume de lisier recueilli semble davantage lié à la consommation d'eau plutôt qu'à un effet des fibres (Levasseur et Texier 2001, Levasseur et Courboulay 2001). Par contre, la composition du lisier est largement modifiée avec une augmentation de sa teneur en matière sèche, celle-ci pouvant être doublée (Levasseur et Courboulay 2001), voire même quintuplée (Massé et al 2003) en comparaison d'un régime à faible teneur en fibres. Cette augmentation est principalement attribuée à la présence dans les fèces de fibres insolubles et d'autres nutriments indigérés. La fraction organique des lisiers est d'ailleurs augmentée en présence de fibres (Levasseur et Courboulay 2001, Massé et al 2003). Le passage d'un lisier liquide à un lisier semi-liquide peut entraîner des problèmes lors de la récolte des effluents ou lors de l'épandage (Robert et al 2000).

La modification de la composition des fèces entraîne un accroissement de la teneur en $\mathrm{AGV}$ et une réduction du pH du lisier (Canh et al 1997, 1998a, 1998b, Massé et al 2003, Hansen et al 2007, Lynch et al 2007). Par exemple, dans le cas d'un aliment à 50\% de NDF (cosses d'avoine, farine de luzerne, son de blé), le lisier produit contient deux fois plus d'AGV (4,9 vs 2,1 g/L) que celui obtenu avec un aliment à $20 \%$ de NDF (maïs, son de blé, orge), le pH étant respectivement de 8,24 et 8,54 avec les deux régimes étudiés (Massé $e t$ al 2003).

La teneur en azote des lisiers semble par contre peu affectée (Canh et al 1998a, Levasseur et Courboulay 2001). En effet, malgré une modification de la répartition des pertes azotées en faveur des fèces et au détriment de l'urine, la quantité totale d'azote rejeté reste constante (Canh et al 1997, Ramonet et al 2000b, Olesen et al 2001, Le Goff et al 2002a, Zervas et Zijltra 2002, Lynch et al 2007). Quant aux contenus des lisiers en minéraux et autres oligo-élé- 
ments, ils sont étroitement liés à la teneur en ces éléments des sources de fibres utilisées et aux éventuels apports complémentaires. Ceci explique que dans certaines études une augmentation de l'excrétion est observée (Levasseur et Courboulay 2001) alors que l'inverse est relaté dans d'autres (Clark et al 2005).

A propos des systèmes d'hébergement sur litière, peu de références bibliographiques sont actuellement disponibles quant à l'effet des fibres sur la composition des fumiers. Des résultats préliminaires (Philippe et al 2007) semblent montrer l'absence d'influence des fibres sur le contenu en MS, en matière organique et sur le $\mathrm{pH}$ de litières de paille accumulée.

\section{2 / Emissions gazeuses}

\section{a) Emissions d'ammoniac}

Plusieurs études menées avec des porcs charcutiers ont montré qu'une ration riche en fibres permettait de réduire les émissions d'ammoniac $\left(\mathrm{NH}_{3}\right)($ Canh et al 1998a, 1998b, Garry et al 2007, Lynch et al 2007). Par exemple, l'incorporation à un régime à base de maïs de $60 \%$ de pulpes de betterave ou de coques de soja permet de réduire les émissions de $25 \%$ et $35 \%$ respectivement (Canh et al 1998b).

L'ammoniac des porcheries est majoritairement issu de l'hydrolyse de l'urée présente dans l'urine, par l'uréase, enzyme produite par les microorganismes des matières fécales (Muck et Steenhuis 1981). Il peut également être formé par dégradation des protéines présentes dans l'effluent. Toutefois, ce processus est lent et ne produit qu'une faible quantité d'ammoniac (Aarnink et al 1993). La formation d'ions ammonium $\left(\mathrm{NH}_{4}{ }^{+}\right)$est essentiellement tributaire de la concentration en urée du lisier et de l'activité de l'uréase (figure 3a). L'équilibre entre $\mathrm{NH}_{4}^{+}$et $\mathrm{NH}_{3 \text { liq }}$ est fonction de la température $\mathrm{du}$ lisier et surtout de son $\mathrm{pH}$ (figure $3 b$ ), une température et un $\mathrm{pH}$ élevés favorisant la fraction non ionisée, $\mathrm{NH}_{3 \text { liq. }}$ Le passage à l'état gazeux (figure $3 c$ ) est fonction de la température, de la concentration du gaz dans le lisier et de la pression partielle du gaz dans l'air (loi de Henry). Enfin, l'émission d'ammoniac dans l'atmosphère dépend principalement de la vitesse de l'air à la surface d'émission (Aarnink et Elzing 1998, Sommer et al 2006).

Dès lors, on peut expliquer l'effet des fibres sur la réduction des émissions d'ammoniac à la fois par la réduction de l'excrétion uréique et par la diminution du $\mathrm{pH}$ du lisier (Canh et al 1998a, 1998b, Garry et al 2007, Hansen et al 2007, Lynch et al 2007). Ainsi des sources de fibres facilement fermentescibles, permettent de réduire les émissions de $\mathrm{NH}_{3}$ en favorisant les fermentations microbiennes, la synthèse d'AGV et la diminution du $\mathrm{pH}$ (Canh et al 1998a, 1998b).

Ces différents résultats obtenus avec des porcs charcutiers devraient pouvoir s'appliquer aux truies gestantes. Massé et al (2003), sans mesurer directement les émissions de $\mathrm{NH}_{3}$, observent une diminution de $\mathrm{pH}$ du lisier lorsque la proportion de fibres dans les rations de truies gestantes augmente. Des études complémentaires mériteraient cependant d'être menées afin de confirmer ces effets. A notre connaissance, il n'existe pas de données équivalentes pour les truies élevées sur litières.

\section{b) Emissions de méthane}

Les émissions de $\mathrm{CH}_{4}$ en porcherie ont deux origines : le tube digestif et l'effluent. La production de $\mathrm{CH}_{4}$ entérique, fonction de la quantité de fibres ingérées, a été discutée dans le paragraphe traitant de la digestion. La production de $\mathrm{CH}_{4}$ à partir de l'effluent provient de la dégradation anaérobique de la matière organique (Hellmann et al 1997). La méthanogenèse est effectuée par une flore mésophile $\left(25-40^{\circ} \mathrm{C}\right)$ à un pH idéal de 7,0 à 7,2. Elle est stimulée par des contenus importants en matière sèche et en matière organique (Hobbs et al 1999. El-Mashad et al 2004, Møller et al 2004, Monteny et al 2006). Les fibres, en augmentant les teneurs en matière sèche et en matière organique et en diminuant le $\mathrm{pH}$ du lisier vers la valeur optimale, favoriseraient donc la capacité de production de méthane de l'effluent (Kirchgessner et al 1991, Aarnink et Verstegen 2007). Cependant, la régulation de sa synthèse au sein de l'effluent est complexe en raison de différentes interactions s'y opérant (Hobbs et al 1999, Monteny et al 2006). Dans les litières, la situation est encore plus complexe. Ainsi, des litières au contenu élevé en eau stimuleraient la production de $\mathrm{CH}_{4}$ en favorisant un environnement anaérobie (Philippe et al 2008).

\section{c) Emissions d'odeurs}

Le et al (2004) ont publié une synthèse sur les relations entre l'alimentation des porcs et la nuisance olfactive. Ils concluent que le rôle des hydrates de carbone fermentescibles sur la production d'odeur dépend à la fois de leur type et des quantités distribuées. Selon ces paramètres, la nuisance olfactive est soit diminuée, soit augmentée, en fonction des populations bactériennes dont le développement est stimulé. Normalement, de par la nature des produits formés, la flore fermentant les hydrates de carbone contribue moins à la nuisance olfactive que la flore protéolytique. Les rations enrichies en fibres devraient donc produire moins d'odeurs mais cette hypothèse n'est pas vérifiée dans tous les cas, ce qui pourrait notamment s'expliquer par des interférences avec le rôle que jouent également la composition et la teneur en protéines des rations sur la formation des composés odorants.

\section{Conclusion}

L'objectif recherché en incorporant des fibres à la ration des truies gestantes est de satisfaire leur appétit sans augmenter la quantité d'énergie ingérée. Avec une teneur en fibres de l'ordre de 15 à $20 \%$ de NDF, une alimentation ad libitum, est envisageable alors qu'avec une ration standard les truies doivent être rationnées, ce qui implique le recours à un dispositif assurant un contrôle individuel des quantités consommées.

En favorisant le rassasiement des truies, ces rations permettent de réduire

Figure 3. Equations impliquées dans la synthèse et l'émission d'ammoniac : (a) formation d'ions ammonium à partir de l'urée, (b) équilibre entre ammonium ionisé et nonionisé, (c) formation d'ammoniac gazeux.

$$
\begin{aligned}
& \mathrm{CO}\left(\mathrm{NH}_{2}\right)_{2}+3 \mathrm{H}_{2} \mathrm{O} \stackrel{\text { uréase }}{\longrightarrow} 2 \mathrm{NH}_{4}{ }^{+}+\mathrm{HCO}_{3^{-}}+\mathrm{OH}^{-} \text {(a) } \\
& \mathrm{NH}_{4}++\mathrm{H}_{2} \mathrm{O} \longleftrightarrow \underbrace{}_{\mathrm{NH}_{3 \mathrm{gaz}}}(\mathrm{c})
\end{aligned}
$$


l'apparition de stéréotypies, comportements anormaux interprétés comme un signe de mal-être des animaux. Les résultats peuvent varier selon la source des fibres, les pulpes de betterave se montrant en général plus efficaces que les fibres d'autres origines (son, sousproduits de brasserie, paille...). Ainsi, un effet bénéfique sur le bien-être des truies peut déjà être observé avec un taux d'incorporation en pulpes de betterave de 15 à $20 \%$. Leurs propriétés physico-chimiques particulières permettent d'expliquer ces résultats, comme par exemple leur grande capacité de liaison à l'eau, qui, en augmentant la viscosité des digesta, ralentit la vidange gastrique.

La présence de fibres va cependant avoir un effet négatif sur la digestibilité de l'énergie des rations en raison de leur taux de lignification qui limite l'accessibilité des enzymes digestives et microbiennes aux composants cellulaires. Cet effet est néanmoins plus limité avec des fibres facilement fermentescibles, riches en cellulose et hémicelluloses et pauvres en lignine, comme celles apportées par les pulpes de betterave. Les AGV issus des fermentations constituent en effet une source d'énergie non-négligeable. En outre, on observe une meilleure valorisation de l'énergie provenant des fibres chez la truie que chez le porc en croissance.

Concernant les performances zootechniques, la plupart des études concluent à l'absence d'effets négatifs des rations riches en fibres sur le gain de poids vif et d'épaisseur de lard dorsal des truies lors de la gestation, la taille et le poids des portées à la naissance, l'intervalle sevrage-fécondation et le taux de conception. L'adaptation du tube digestif à la consommation de plus grandes quantités d'aliments augmente l'ingestion en début de lactation ce qui limite la mobilisation des réserves corporelles et soutient la production laitière.

La quantité d'effluent récolté sous forme de lisier n'est pas modifiée, une augmentation de la production de matières fécales étant compensée par une diminution de la production d'urine. Les émissions d'ammoniac à partir des lisiers sont diminuées car une partie de l'azote de l'urée plasmatique est incorporée dans les protéines de la flore intestinale et le $\mathrm{pH}$ des matières fécales est abaissé suite à la production d'AGV. La réduction des émissions d'ammoniac est d'ailleurs d'autant plus importante que les fibres sont fermentescibles. A l'inverse, les émissions de méthane semblent favorisées par le $\mathrm{pH}$ bas et les teneurs élevées des lisiers en matière sèche et matière organique.

\section{Références}

Aarnink A.J.A., Elzing A., 1998. Dynamic model for ammonia volatilization in housing with partially slatted floors, for fattening pigs. Livest. Prod. Sci., 53, 153-169.

Aarnink A.J.A., Verstegen M.W.A., 2007. Nutrition, key factor to reduce environmental load from pig production. Livest. Sci., 109, 194-203.

Aarnink A.J.A., Hoeksma P., van Ouwerkerk E.N.J., 1993. Factors affecting ammonium concentration in slurry from fattening pigs. Nitrogen flow in pig production and environmental consequences. Proc. $1^{\text {st }}$ Int. Symp., Wageningen, The Netherlands, 413-420.

Anguita M., Gasa J., Nofrarias M., MartinOrue S.M., Perez J.F., 2007. Effect of coarse ground corn, sugar beet pulp and wheat bran on the voluntary intake and physicochemical characteristics of digesta of growing pigs. Livest. Sci., 107, 182-191.

Bach Knudsen K.E., 1997. Carbohydrate and lignin contents of plant materials used in animal feeding. Anim. Feed Sci. Technol., 67, 319-338.

Bach Knudsen K.E., 2001. The nutritional significance of «dietary fibre» analysis. Anim. Feed Sci. Technol., 90, 3-20.

Bergeron R., Bolduc J., Ramonet Y., MeunierSalaün M.C., Robert S., 2000. Feeding motivation and stereotypies in pregnant sows fed increasing levels of fibre and/or food. Applied Anim. Behav. Sci., 70, 27-40.

Bergner H., 1981. Chemically treated straw meal as a new source of fibre in the nutrition of pigs. Pig News Inf., 2, 135-140.

Bertin C., Rouau X., Thibault J.F., 1988. Structure and properties of sugar-beet fibers. J. Sci. Food Agric., 44, 15-29.

Bilkei G.P., 1990. The effect of increased fiber content fed on the previous week on the parturition of sows. Magyar Allatorvosok Lapja, 45, 597-601.
Bindelle J., Buldgen A., Wavreille J., Agneessens R. Destain J.P., Wathelet B. Leterme P., 2007. The source of fermentable carbohydrates influences the in vitro protein synthesis by colonic bacteria isolated from pigs. Animal, 1, 1126-1133.

Broom D., 1988. Sow welfare indicators. Vet. Rec., 123, 235-235.

Brouns F., Edwards S.A., English P.R., 1994a. Effect of dietary fiber and feeding system on activity and oral behavior of group-housed gilts. Applied Anim. Behav. Sci., 39, 215-223.

Brouns F., Edwards S.A., English P.R., 1994b. Metabolic effects of fibrous ingredients in pig diets. Anim. Prod., 58, 467

Brouns F., Edwards S.A., English P.R., 1995. Influence of fibrous feed ingredients on voluntary intake of dry sows. Anim. Feed Sci. Technol., 54, 301-313.

Brouns F., Edwards S.A., English P.R., 1997. The effect of dietary inclusion of sugar-beet pulp on the feeding behaviour of dry sows. Anim. Sci., 65, 129-133.

Canh T.T., Verstegen M.W.A., Aarnink A.J.A., Schrama J.W., 1997. Influence of dietary factors on nitrogen partitioning and composition of urine and feces of fattening pigs. J. Anim. Sci., $75,700-706$

Canh T.T., Schrama J.W., Aarnink A.J.A., Verstegen M.W.A., van't Klooster C.E., Heetkamp M.J.W., 1998a. Effect of dietary fermentable fibre from pressed sugar-beet pulp silage on ammonia emission from slurry of growing-finishing pigs. Anim. Sci., 67, 583590.

Canh T.T., Sutton A.L., Aarnink A.J.A., Verstegen M.W.A., Schrama J.W., Bakker G.C.M., 1998b. Dietary carbohydrates alter the fecal composition and $\mathrm{pH}$ and the ammonia emission from slurry of growing pigs. J. Anim. Sci., 76, 1887-1895
Cerneau P., Meunier-Salaun M.C., Lauden P., Godfrin K., 1997. Incidence du mode de logement et du mode d'alimentation sur le comportement de truies gestantes et leurs performances de reproduction. Journ. Rech. Porcine Fr., 29, 175 182.

Cherbut C., Barry J.L., Wyers M., Delortlaval J., 1988. Effect of the nature of dietary fiber on transit-time and fecal excretion in the growingpig. Anim. Feed Sci. Technol., 20, 327-333.

Clark O.G., Moehn S., Edeogu I., Price J., Leonard J., 2005. Manipulation of dietary protein and nonstarch polysaccharide to control swine manure emissions. J. Env. Quality, 34, 1461-1466.

Close W.H., Cole D.J.A., 1986. Some aspects of the nutritional-requirements of sows - their relevance in the development of a feeding strategy. Livest. Prod. Sci., 15, 39-52.

Close W.H., Noblet J., Heavens R.P., 1984. The partition of body-weight gain in the pregnant sow. Livest. Prod. Sci., 11, 517-527.

Courboulay V., Gaudre D., 2002. Faut-il distribuer des aliments enrichis en fibres aux truies en groupe ? Journ. Rech. Porcine Fr., Paris, France, 34, 225-232.

Cuche G., Cuber J.C., Malbert C.H., 2000. Ileal short-chain fatty acids inhibit gastric motility by a humoral pathway. Am. J. Physiol., Gastrointestinal and Liver Physiology, 279, G925-G930.

Danielsen V., Vestergaard E.M., 2001. Dietary fibre for pregnant sows: effect on performance and behaviour. Anim. Feed Sci. Technol., 90, 71-80.

Dantzer R., 1986. Behavioral, physiological and functional aspects of stereotyped behavior: a review and a re-interpretation. J. Anim. Sci., 62, 1776-1786.

de Leeuw J.A., Jongbloed A.W., Spoolder H.A.M., Verstegen M.W.A., 2005. Effects of 
hindgut fermentation of non-starch polysaccharides on the stability of blood glucose and insulin levels and physical activity in empty sows. Livest. Prod. Sci., 96, 165-174.

Deplancke B., Gaskins H.R., 2001. Microbial modulation of innate defense: goblet cells and the intestinal mucus layer. Am. J. Clin. Nutr., 73, 1131S-1141S.

Dierick N.A., Vervaeke I.J., Demeyer D.I., Decuypere J.A., 1989. Approach to the energetic importance of fiber digestion in pigs. 1. Importance of fermentation in the overall energy supply. Anim. Feed Sci. Technol., 23, 141-167.

Dourmad J.Y., 1991. Effect of feeding level in the gilt during pregnancy on voluntary feed-intake during lactation and changes in body-composition during gestation and lactation. Livest. Prod. Sci., 27, 309-319.

Dourmad J.Y., Etienne M., Noblet J., 1994. La reconstitution des réserves corporelles chez la truie multipare en gestation : influence du niveau de mobilisation au cours de la lactation précédente. Journ. Rech. Porcine Fr., Paris, France, $26,277-283$.

Eissen J.J., Apeldoorn E.J., Kanis E., Verstegen M.W.A., de Greef K.H., 2003. The importance of a high feed intake during lactation of primiparous sows nursing large litters. J. Anim. Sci., 81, 594-603.

El-Mashad H.M., Zeeman G., van Loon W.K.P., Bot G.P.A., Lettinga G., 2004. Effect of temperature and temperature fluctuation on thermophilic anaerobic digestion of cattle manure. Bioresource Technology, 95, 191-201.

Farmer C., Robert S., Matte J.J., 1996. Lactation performance of sows fed a bulky diet during gestation and receiving growth hormonereleasing factor during lactation. J. Anim. Sci., 74, 1298-1306.

Fernandez J.A., 2006. The composition of faeces and urine from slaughter pigs and gestating sows is determined by diet composition. DIAS Report, Plant Prod., 85-87.

Fraser D., 1975. The effect of straw on the behaviour of sows in tether stalls. Anim. Prod., $21,59-68$.

Fukunaga T., Sasaki M., Araki Y., Okamoto T., Yasuoka T., Tsujikawa T., Fujiyama Y., Bamba T., 2003. Effects of the soluble fibre pectin on intestinal cell proliferation, fecal short chain fatty acid production and microbial population. Digestion, 67, 42-49.

Garry B.P., Fogarty M., Curran T.P., O'Connell M.J., O'Doherty J.V., 2007. The effect of cereal type and enzyme addition on pig performance, intestinal microflora, and ammonia and odour emissions. Animal, 1, 751-757.

Guérin S., Ramonet Y., LeCloarec J., Meunier-Salaun M.C., Bourguet P., Malbert C.H., 2001. Changes in intragastric meal distribution are better predictors of gastric emptying rate in conscious pigs than are meal viscosity or dietary fibre concentration. Br. J. Nutr., 85, 343350 .

Guillemet R., Dourmad J.Y., Meunier-Salaun M.C., 2006. Feeding behavior in primiparous lactating sows: impact of a high-fiber diet during pregnancy. J. Anim. Sci., 84, 2474-2481.

Guillemet R., Comyn S., Dourmad J.Y., Meunier-Salaun M.C., 2007a. Gestating sows prefer concentrate diets to high-fibre diet in twochoice tests. Applied Anim. Behav. Sci., 108, 251-262.
Guillemet R., Hamard A., Quesnel H., Pere M.C., Etienne M., Dourmad J.Y., MeunierSalaun M.C., 2007b. Dietary fibre for gestating sows: effects on parturition progress, behaviour, litter and sow performance. Animal, 1, 872-880.

Hansen M.J., Chwalibog A., Tauson A.H., 2007. Influence of different fibre sources in diets for growing pigs on chemical composition of faeces and slurry and ammonia emission from slurry. Anim. Feed Sci. Technol., 134, 326-336.

Hellmann B., Zelles L., Palojarvi A., Bai Q.Y., 1997. Emission of climate-relevant trace gases and succession of microbial communities during open-window composting. Applied Env. Microbiol., 63, 1011-1018.

Hobbs P.J., Misselbrook T.H., Cumby T.R. 1999. Production and emission of odours and gases from ageing pig waste. J. Agric. Eng. Res., $72,291-298$

Holt J.P., Johnston L J., Baidoo S.K., Shurson G.C., 2006. Effects of a high-fiber diet and frequent feeding on behavior, reproductive performance, and nutrient digestibility in gestating sows. J. Anim. Sci., 84, 946-955.

Honeyman M.S., Zimmerman D.R., 1990. Long-term effects of corn gluten feed on the reproductive-performance and weight of gestating sows. J. Anim. Sci., 68, 1329-1336.

Jin L., Reynolds L.P., Redmer D.A., Caton J.S., Crenshaw J.D., 1994. Effects of dietary fiber and on intestinal growth, cell-proliferation, morphology in growing pigs. J. Anim. Sci., 72, 2270-2278.

Jørgensen H., 2007. Methane emission by growing pigs and adult sows as influenced by fermentation. Livest. Sci., 109, 216-219.

Jørgensen H., Zhao X.Q., Eggum B.O., 1996. The influence of dietary fibre and environmental temperature on the development of the gastrointestinal tract, digestibility, degree of fermentation in the hind-gut and energy metabolism in pigs. Br. J. Nutr., 75, 365-378.

Jørgensen H., Serena A., Hedemann M.S., Knudsen K.E.B., 2007. The fermentative capacity of growing pigs and adult sows fed diets with contrasting type and level of dietary fibre. Livest. Sci., 109, 111-114.

Kirchgessner M., Kreuzer M., Muller H.L., Windisch W. 1991. Release of methane and of carbon-dioxide by the pig. Agribiological Research-Zeitschrift Fur Agrarbiologie Agrikulturchemie Okologie, 44, 103-113.

Kurcman-Przedpelska B., 1989. Duration of pregnancy and farrowing of sows fed on concentrate mixtures of different fibre content. Acta Acad. Agricult. Techn. Olstenensis, Zootechn., $32,65-74$.

Latymer E.A., Low A.G., Fadden K., Sambrook I.E., Woodley S.C., Keal H.D., 1990. Measurement of transit-time of digesta through sections of gastrointestinal-tract of pigs fed with diets containing various sources of dietary fiber (nonstarch polysaccharides). Arch. Tierernahr., Archiv. Anim. Nutr., 40, 667-680.

Le P.D., Becker P.M., Aarnink A.J.A., Jongbloed A.W., van der Peet-Schwering C.M.C., 2004. Odour from pig production facilities: Its relation to diet. Agrotechnology and food innovations B.V., Wageningen, Pays-Bas, Report $115,65 \mathrm{p}$.

Le Cozler Y., Neil M., Ringmar-Cederberg E., Dourmad J.Y., 1999. Effet du niveau d'alimentation pendant la croissance et de la stratégie de mise à la reproduction sur les performances des truies au cours des deux premiers cycles de reproduction. Journ. Rech. Porcine Fr., Paris, France, 31, 9-15.

Le Goff G., 2001. Etude des mécanismes impliqués dans l'évolution de la digestion et l'utilisation métabolique des fibres alimentaires au cours de la vie du porc. Conséquences pour l'estimation de la valeur énergétique des aliments. Thèse, Ecole nationale supérieure agronomique de Rennes, Rennes, France, 270p.

Le Goff G., Noblet J., 2001. Comparative total tract digestibility of dietary energy and nutrients in growing pigs and adult sows. J. Anim. Sci., $79,2418-2427$.

Le Goff G., Le Groumellec L., van Milgen J., Dubois S., Noblet J., 2002a. Digestibility and metabolic utilisation of dietary energy in adult sows: influence of addition and origin of dietary fibre. Br. J. Nutr., 87, 325-335.

Le Goff G., van Milgen J., Noblet J., 2002b. Influence of dietary fibre on digestive utilization and rate of passage in growing pigs, finishing pigs and adult sows. Anim. Sci., 74, 503-515.

Le Goff G., Noblet J., Cherbut C., 2003. Intrinsic ability of the faecal microbial flora to ferment dietary fibre at different growth stages of pigs. Livest. Prod. Sci., 81, 7587.

Levasseur P., Courboulay V., 2001. Quels rejets pour des truies gestantes alimentées avec un régime enrichi en fibres ? Techni-Porc, 24, 13-16.

Levasseur P., Texier C., 2001. Teneurs en éléments - traces métalliques des aliments et des lisiers de porcs à l'engrais, de truies et de porcelets. Journ. Rech. Porcine Fr., Paris, France, 33, 57-62.

Libao-Mercado A.J, Zhu C.L, Fuller M.F Rademacher M., Seve B., de Lange C.F.M., 2007. Effect of feeding fermentable fiber on synthesis of total and mucosal protein in the intestine of the growing pig. Livest. Sci., 109, 125-128.

Low A.G., 1985. Role of dietary fibre in pig diet, In: Recent advances in animal nutrition, Haresign W., Cole D.J.A. (Eds), Butterworths, London, UK, 87.

Lynch M.B., Sweeney T., Callan J.J., O'Doherty J.V., 2007. The effect of dietary barley level on volatile fatty acid concentration and manure ammonia emissions in finishing pigs. Livest. Sci., 109, 236-239.

Lynch M.B., O'Shea C.J., Sweeney T., Callan J.J., O'Doherty J.V. 2008. Effect of crude protein concentration and sugar-beet pulp on nutrient digestibility, nitrogen excretion, intestinal fermentation and manure ammonia and odour emissions from finisher pigs. Animal, 2, 425434.

Martin J.E., Edwards S.A., 1994. Feedingbehavior of outdoos sows - The effects of diet quantity and type. Applied Anim. Behav. Sci., 41, 63-74.

Massé D.I., Croteau F., Masse L., Bergeron R., Bolduc J., Ramonet Y., Meunier-Salaun M.C., Robert S., 2003. Effect of dietary fiber incorporation on the characteristics of pregnant sows slurry. Can. Biosystems Eng., 45, 6.7-6.12.

Matte J.J., Robert S., Girard C.L., Farmer C., Martineau G.P., 1994. Effect of bulky diets based on wheat bran or oat hulls on reproductive-performance of sows during their first 2 parities. J. Anim. Sci., 72, 1754-1760.

Meunier-Salaün M.C., Edwards S.A., Robert S., 2001. Effect of dietary fibre on the behaviour 
and health of the restricted fed sow. Anim. Feed Sci. Technol., 90, 53-69.

Miquel N., Bach Knudsen K.E., Jørgensen H., 2001. Impact of diets varying in dietary fibre characteristics on gastric emptying in pregnant sows. Arch. Anim. Nutr., Arch. Tierernahrung, $55,121-145$.

Møller H.B., Sommer S.G., Ahring B., 2004. Methane productivity of manure, straw and solid fractions of manure. Biomass and Bioenergy, 26, 485-495.

Monteny G.J., Bannink A., Chadwick D., 2006. Greenhouse gas abatement strategies for animal husbandry. Agric. Ecosyst. Env., 112, 163-170.

Moran T.H., Wirth J.B., Schwartz G.J., McHugh P.R., 1999. Interactions between gastric volume and duodenal nutrients in the control of liquid gastric emptying. Am. J. Physiol., Regulatory Integrative and Comparative Physiology, 45, R997-R1002.

Morgenthum R., Bolduan G., 1988. Effects of live weight and dietary factors of sows on length of parturition. Monatshefte Fur Veterinarmedizin, 43, 194-196.

Mosenthin R., Sauer W.C., Ahrens F., 1994. Dietary pectins effect on ileal and fecal aminoacid digestibility and exocrine pancreatic secretions in growing pigs. J. Nutr., 124, 1222-1229.

Mroz Z., Partridge I.G., Mitchell G., Keal H.D., 1986. The effect of oat hulls, added to the basal ration for pregnant sows, on reproductiveperformance, apparent digestibility, rate of passage and plasma parameters. J. Sci. Food Agric. $37,239-247$

Muck R.E., Steenhuis T.S., 1981. Nitrogen losses in free stall dairy barns, In: Engineering, A.S.O.A. (Ed.), Livestock waste: a renewable resource, American Society of Agriculture Engineering, St Joseph, Michigan, USA, 406-409.

Noblet J., Le Goff G., 2000. Utilisation digestive et valeurs énergétiques du blé, du maïs et de leurs coproduits chez le porc en croissance et la truie adulte. Journ. Rech. Porcine Fr., Paris, France, 32, 177-183.

Noblet J., Dourmad J.Y., Ledividich J., Dubois S., 1989. Effect of ambient-temperature and addition of straw or alfalfa in the diet on energy-metabolism in pregnant sows. Livest. Prod. Sci., 21, 309-324.

Noblet J., Shi X. S., Dubois,S., 1994. Composantes de la dépense énergétique au cours du nycthémère chez la truie adulte à l'entretien : rôle de l'activité physique. INRA Prod. Anim., 7, 135-142.

Noblet J., Bontems V., Tran G., 2003. Estimation de la valeur énergétique des aliments pour le porc. INRA Prod. Anim., 16, 197-210.

Olesen C.S., Jørgensen H., Danielsen V., 2001. Effect of dietary fibre on digestibility and energy metabolism in pregnant sows. Acta Agric. Scand., A, Animal Science, 51, 200-207.

Paboeuf F., Ramonet Y., Corlouer A., Dourmad J.Y., Cariolet R., Meunier-Salaun M.C., 2000. Impact de l'incorporation de fibres dans un régime de gestation sur les performances zootechniques et le comportement des truies. Journ. Rech. Porcine Fr., Paris, France, 32, 105-113.

Partanen K., Jalava T., Valaja I., 2007. Effects of a dietary organic acid mixture and of dietary fibre levels on ileal and faecal nutrient apparent digestibility, bacterial nitrogen flow, microbial metabolite concentrations and rate of passage in the digestive tract of pigs. Animal, 1, 389-401.
Patience J.F., Austic R.E., Boyd R.D., 1987. Effect of dietary electrolyte balance on growth and acid-base status in swine. J. Anim. Sci., 64, 457-466.

Philippe F.X., Zizo A., Canart B., Laitat M. Vandenheede M., Wavreille J., Bartiaux-Thill N., Nicks B., 2007. Evaluation environnementale de la technique d'élevage des truies en groupe sur litière, $3^{\text {ème }}$ rapport intermédiaire. Liège, $30 \mathrm{p}$.

Philippe F.X., Canart B., Laitat M. Vandenheede M., Wavreille J., Bartiaux-Thill N., Nicks B., 2008. Effet des consommations alimentaires sur les émissions gazeuses associées à l'élevage de truies gestantes en groupe sur litière. Journ. Rech. Porcine Fr., Paris, France, 40, 263 266

Plaisancie P., Dumoulin V., Chayvialle J.A., Cuber J.C., 1996. Luminal peptide YY-releasing factors in the isolated vascularly perfused rat colon. J. Endocrinol., 151, 421-429.

Pollmann D.S., Danielson D.M., Peo E.R., 1979. Value of high-fiber diets for gravid swine. J. Anim. Sci., 48, 1385-1393.

Potkins Z.V., Lawrence T.L.J., Thomlinson J.R., 1991. Effects of structural and nonstructural polysaccharides in the diet of the growing pig on gastric-emptying rate and rate of passage of digesta to the terminal ileum and through the total gastrointestinal-tract. Br. J. Nutr., 65, 391413.

Prosky L., Asp N.G., Schweizer T.F., Devries J., Furda I., 1988. Determination of insoluble soluble, and total dietary fiber in foods and foodproducts - Interlaboratory study. J. Ass. Off. Analytical Chem., 71, 1017-1023.

Prunier A., Dourmad J.Y., Etienne M., 1993. Feeding level, metabolic parameters and reproductive-performance of primiparous sows. Livest. Prod. Sci., 37, 185-196.

Quesnel H., Etienne M., Pere M. C., 2007. Influence of litter size on metabolic status and reproductive axis in primiparous sows. J. Anim. Sci., $85,118-128$.

Ramonet Y., Meunier-Salaun M.C., Dourmad J.Y., 1997. Effets d'une incorporation de parois végétales dans la ration alimentaire sur l'activité comportementale des truies gestantes. Journ. Rech. Porcine Fr., Paris, France, 29, 167-174.

Ramonet Y., Meunier-Salaun M.C., Dourmad J.Y., 1999. High-fiber diets in pregnant sows: Digestive utilization and effects on the behavior of the animals. J. Anim. Sci., 77, 591-599.

Ramonet Y., Robert S., Aumaitre A., Dourmad J.Y., Meunier-Salaun M.C., 2000a. Influence of the nature of dietary fibre on digestive utilization, some metabolite and hormone profiles and the behaviour of pregnant sows. Anim. Sci., 70, 275-286.

Ramonet Y., van Milgen J., Dourmad J.Y., Dubois S., Meunier-Salaun M.C., Noblet J., 2000 b. The effect of dietary fibre on energy utilisation and partitioning of heat production over pregnancy in sows. Br. J. Nutr., 84, 85-94.

Rijnen M.M.J.A., Verstegen M.W.A., Heetkamp M.J.W., Haaksma J., Schrama J.W., 2001. Effects of dietary fermentable carbohydrates on energy metabolism in group-housed sows. J. Anim. Sci., 79, 148-154.

Rijnen M.M.J.A., Verstegen M.W.A., Heetkamp M.J.W., Haaksma J., Schrama J.W. 2003. Effects of dietary fermentable carbohydrates on behavior and heat production in grouphoused sows. J. Anim. Sci., 81, 182-190.
Robert S., Matte J.J., Farmer C., Girard C.L., Martineau G.P., 1993. High fiber diets for sows Effects on stereotypies and adjunctive drinking Applied Anim. Behav. Sci., 37, 297-309.

Robert S., Rushen J., Farmer C., 1997. Both energy content and bulk of food affect stereotypic behaviour, heart rate and feeding motivation of female pigs. Applied Anim. Behav. Sci., 54, 161-171.

Robert S., Farmer C., Rushen J., 2000. Manure production of first-litter sows fed a highfibre diet during gestation. Can. J. Anim. Sci. 80, 737-739.

Robert S., Bergeron R., Farmer C., MeunierSalaun M.C., 2002. Does the number of daily meals affect feeding motivation and behaviour of gilts fed high-fibre diets? Applied Anim. Behav. Sci., 76, 105-117.

Salvador V., Cherbut C., 1992. Regulation du transit digestif par les fibres alimentaires. Cah. Nutr. Diététique, 27, 290-297.

Satchithanandam S., Vargofcakapker M., Calvert R.J., Leeds A.R., Cassidy M.M., 1990. Alteration of gastrointestinal mucin by fiber feeding in rats. J. Nutr., 120, 1179-1184.

Sauvant D., Perez J.M., Tran G., 2004. Tables de composition et de valeur nutritive des matières premières destinées aux animaux d'élevage porcs, volailles, bovins, ovins, caprins, lapins, chevaux, poissons. 2ème Edition revue et corrigée. INRA Editions, Paris, France, 301p.

Serena A., Hedemann M.S., Knudsen K.E.B. 2007. Feeding high fibre diets changes luminal environment and morphology in the intestine of sows. Livest. Sci., 109, 115-117.

Shi X.S., Noblet J., 1993. Contribution of the hindgut to digestion of diets in growing pigs and adult sows. Effect of diet composition. Livest. Prod. Sci., 34, 237-252.

Smith E.A., Macfarlane G.T., 1997 Dissimilatory amino acid metabolism in human colonic bacteria. Anaerobe, 3, 327-337.

Sommer S.G., Zhang G.Q., Bannink A., Chadwick D., Misselbrook T., Harrison R., Hutchings N.J., Menzi H., Monteny G.J., Ni J.Q., Oenema O., Webb J., 2006. Algorithms determining ammonia emission from buildings housing cattle and pigs and from manure stores. Adv. Agron., 89, 261-335.

Svendsen J., Svendsen L.S., 1997. Intensive (commercial) systems for breeding sows and piglets to weaning. Livest. Prod. Sci., 49, 165-179.

Terlouw E.M.C., Lawrence A.B., Illius A.W., 1991. Influences of feeding level and physical restriction on development of stereotypies in sows. Anim. Behav., 42, 981-991.

Thibault J.F., Renard C.M.J., Guillon F. 1994. Physical and chemical analysis of dietary fibres in sugar beet and vegetables. Modern Methods of Plant Analysis, 14, 23-55.

van der Peet-Schwering C.M.C., Kemp B., Binnendijk G.P., den Hartog L.A., Spoolder H.A.M., Verstegen M.W.A., 2003a. Performance of sows fed high levels of nonstarch polysaccharides during gestation and lactation over three parities. J. Anim. Sci., 81, 2247-2258.

van der Peet-Schwering C.M.C., Spoolder H.A.M., Kemp B., Binnendijk G.P., den Hartog L.A., Verstegen M.W.A., 2003b. Development of stereotypic behaviour in sows fed a starch diet or a non-starch polysaccharide diet during gestation and lactation over two parities. Applied Anim. Behav. Sci., 83, 81-97. 
van der Peet-Schwering C.M.C., Kemp B., Plagge J.G., Vereijken P.F.G., den Hartog L.A., Spoolder H.A.M., Verstegen M.W.A., 2004. Performance and individual feed intake characteristics of group-housed sows fed a nonstarch polysaccharides diet ad libitum during gestation over three parities. J. Anim. Sci., 82, 1246-1257.

van Dijk A.J., van Rens B., van der Lende T., Taverne M.A.M., 2005. Factors affecting duration of the expulsive stage of parturition and piglet birth intervals in sows with uncomplicated, spontaneous farrowings. Theriogenology, 64, 1573-1590.

van Putten G., van de Burgwal J.A., 1990. Vulva biting in group-housed sows: preliminary report. Applied Anim. Behav. Sci., 26, 181-186.

Van Soest P.J., 1967. Development of a comprehensive system of feed analyses and its application to forages. J. Anim. Sci., 26, 119-128.

Varel V.H., Yen J.T. 1997. Microbial perspective on fiber utilization by swine. J. Anim. Sci., 75, 2715-2722.

Vestergaard E.M., Danielsen V., 1998. Dietary fibre for sows: effects of large amounts of soluble and insoluble fibres in the pregnancy period on the performance of sows during three reproductive cycles. Anim. Sci., 67, 355-362.
Warpechowski M.B., van Milgen J., Dubois S., Noblet J., 2007. Producão de calor e produção de metano por porcas sob dietas com deifferentes níveis de fibra. $44^{\mathrm{a}}$ Reunião Anual da Sociedade Brasileira de Zootecnia, UnespJaboticabal, 24-27 de Julho.

Wenk C., 2001. The role of dietary fibre in the digestive physiology of the pig. Anim. Feed Sci. Technol., 90, 21-33.

Whittaker X., Edwards S.A., Spoolder HAM., Lawrence A.B., Corning S., 1995. The effect of ad libitum feeding of a high fibre diet on aggressive interactions in pregnant sows following dynamic grouping. Proceedings of the Winter Meeting, ISAE, London.

Whittaker X., Spoolder H.A.M., Edwards S.A., Lawrence A.B., Corning S., 1998. The influence of dietary fibre and the provision of straw on the development of stereotypic behaviour in food restricted pregnant sows. Applied Anim. Behav. Sci., 61, 89-102.

Whittaker X., Edwards S.A., Spoolder H.A.M., Lawrence A.B., Corning S., 1999. Effects of straw bedding and high fibre diets on the behaviour of floor fed group-housed sows. Applied Anim. Behav. Sci., 63, 25-39.

Whittaker X., Edwards S.A., Spoolder H.A.M., Corning S., Lawrence A.B., 2000. The performance of group-housed sows offered a high fibre diet ad libitum. Anim. Sci., 70, 85-93.

Wiepkema P.R., Broom D.M., Duncan I.J.H., Van Putten G., 1983. Abnormal behaviours in farm animals. Commission of the European Communities Report, Brussels, 16.

Wilfart A., Montagne L., Simmins H., Noblet J., van Milgen J., 2007. Effect of fibre content in the diet on the mean retention time in different segments of the digestive tract in growing pigs. Livest. Sci., 109, 27-29.

Yan, T., Longland, A.C., Close, W.H., Sharpe, C.E., Keal, H.D., 1995. The digestion of drymatter and nonstarch polysaccharides from diets containing plain sugar-beet pulp or wheat-straw by pregnant sows. Anim. Sci., 61, 305-309.

Zebrowska T., Low A.G., Zebrowska H. 1983. Studies on gastric digestion of protein and carbohydrate, gastric-secretion and exocrine pancreatic-secretion in the growing pig. Br. J. Nutr., 49, 401-410.

Zervas S., Zijlstra R.T., 2002. Effects of dietary protein and fermentable fiber on nitrogen excretion patterns and plasma urea in grower pigs. J. Anim. Sci., 80, 3247-3256.

\section{Résumé}

L'intérêt premier d'alimenter les truies gestantes avec des rations enrichies en fibres (REF) est de les rassasier sans apport excessif d'énergie. Une teneur en fibres de l'ordre de 15-20\% de NDF (Neutral Detergent Fibre) permet ainsi aux truies d'adapter leur consommation à leurs besoins, ce qui permet d'envisager une distribution à volonté des aliments, technique qui réduit notablement le coût de l'équipement d'alimentation. L'effet des fibres sur la sensation de satiété est notamment attribuable aux plus grandes quantités consommées, à l'augmentation du temps consacré à l'ingestion et à un ralentissement de la vidange gastrique. L'amélioration du niveau de bienêtre des truies résultant du rassasiement se traduit par une réduction de la fréquence des stéréotypies et des agressions. L'inconvénient d'une distribution de REF tient à une diminution, cependant limitée, de la digestibilité de l'énergie associée à une accélération du transit intestinal, une production accrue de méthane lors des fermentations et une diminution de l'accessibilité des enzymes digestives et microbiennes aux composants cellulaires. La valorisation de l'énergie provenant des fibres chez la truie est cependant meilleure que chez le porc en croissance. Pour autant que les apports alimentaires tiennent compte de la réduction de la digestibilité de l'énergie, les performances de reproduction des truies ne sont pas affectées. Au contraire, le développement du volume du tube digestif induit par les REF en cours de gestation permet une augmentation de l'ingestion en début de lactation, favorisant ainsi la production laitière et limitant la mobilisation des réserves corporelles. Au niveau environnemental, le recours aux REF diminue les émissions d'ammoniac mais favorise les émissions de méthane.

\section{Abstract}

\section{Food fibers in gestating sows: effects on nutrition, behaviour, performances and waste in the environment}

Feeding gestating sows with high-fibre diets (HFD) has as a major interest to induce satiety without excessive energy intake. NDF (Neutral Detergent Fibre) fibre content (15-20\%) enables sows to adapt their feed intake to their needs. Therefore, ad libitum feeding becomes possible, reducing the cost of feeding equipment. Fibre effect on the feeling of satiety is due, among others, to the higher amounts of feed intake, the increase of eating time and the delay in gastric emptying. Sows welfare improvement due to the feeling of satiety has for consequence a reduction in the occurrence of stereotypes and aggressions. The disadvantage of HFD feeding is linked to a reduction, however limited, in the energy digestibility associated with an increase in the transit speed, a higher methane production and a reduction of cell component accessibility to digestive and microbial enzymes. However, energy efficiency of fibre degradation is better for sows than for growing pigs. If the amount of feed offered takes into account the reduction in energy digestibility, sow performance is not affected. Besides, the increased capacity of the digestive tract may result in an increased feed intake during early lactation, supporting higher milk production and limiting the body reserve mobilisation. On the environmental level, increasing fibre content reduces ammonia emissions but increases methane emissions.

PHILIPPE F.-X., REMIENCE V., DOURMAD J.-Y., CABARAUX J.-F., VANDENHEEDE M., NICKS B., 2008. Les fibres dans l'alimentation des truies gestantes : effets sur la nutrition, le comportement, les performances et les rejets dans l'environnement. INRA Prod. Anim., 21, 277-290. 